\title{
Ruta integral de atención en salud para personas con amputaciones de miembro inferior, para mejorar el funcionamiento y la calidad de vida
}

\author{
Integral pathway of health care for people with lower limb amputations, to improve \\ the functioning and quality of life.
}

\author{
Rota abrangente de cuidados de saúde de pessoas com amputações de \\ membros inferiores, para melhorar o funcionamento e qualidade de vida.
}

Laura Giraldo Castaño; ; Julieth Katerine Pinto Maquilón²; Luz Helena Lugo-Agudelo³; Juan Carlos Velásquez Correa4; María del Pilar Pastor5; Ana María Posada Borrero6; Daniel Felipe Patiño-Lugo7; Jesús Alberto Plata Contreras ${ }^{8}$

1 Médica fisiatra, Universidad de Antioquia, Medellín, Colombia. laura.giraldoc1@udea.edu.co. orCID: https://orcid.org/0000-00029587-4610

2 Médica fisiatra, Universidad de Antioquia, Medellín, Colombia. julieth.pintom@udea.edu.co. oRCID: https://orcid.org/0000-0002-7042-4867

3 MSc en Epidemiología Clínica, Universidad de Antioquia, Medellín, Colombia. luzh.lugo@gmail.com. ORcid: https://orcid.org/00000002-3467-8835

4 Especialista en Estadística, Universidad de Antioquia, Medellín, Colombia. jucaveca11@gmail.com. ORCID: https://orcid.org/00000002-0490-0933

5 Doctorada en Ciencias de la Salud Pública, Universidad de Antioquia, Medellín, Colombia. maria.pastor@udea.edu.co. orcid: https:// orcid.org/0000-0002-9555-3286

$6 \quad$ MSc en Epidemiología Clínica, Universidad de Antioquia, Colombia. amposada@gmail.com. orcid: https://orcid.org/0000-0002-0727-3269

7 PhD en Políticas de Salud, Universidad de Antioquia, Medellín, Colombia. felipe.patino@udea.edu.co. orcid: https://orcid.org/00000003-4030-4255

8 Maestría en Ciencias Clínicas, Universidad de Antioquia, Medellín, Colombia. jesus.plata@udea.edu.co. ORCID: https://orcid.org/00000003-0284-092X

Recibido: 13/08/2020. Aprobado: 26/08/2021. Publicado: 25/01/2022

Giraldo Castaño L, Pinto-Maquilón JK, Lugo-Agudelo LH, Velásquez-Correa JC, Pastor MP, Posada-Borrero AM, Patiño-Lugo DF, Plata-Contreras JA. Ruta integral de atención en salud para personas con amputaciones de miembro inferior, para mejorar el funcionamiento y la calidad de vida. Rev. Fac. Nac. Salud Pública. 2022;40(1):e342981. doi: https://doi.org/10.17533/udea. rfnsp.e342981

\section{Resumen}

Objetivo: Presentar el estudio mediante el cual se construyó una ruta integral de atención en salud (RIAS) para la atención del paciente amputado de miembro inferior por causas traumática, vascular o diabetes mellitus, cuyo fin es implementar las recomendaciones de la Guía de práctica clínica del paciente amputado y garantizar la atención integral en salud de esta población en Colombia. Con la ruta se pretende orientar a los actores involucrados en la ejecución de intervenciones 
individuales para el diagnóstico, el tratamiento y la rehabilitación, e impactar en los desenlaces en salud y equidad de esta población. Metodología: Este estudio responde a una estrategia de mejoramiento de la atención en salud. Para esto, se revisó el Manual metodológico para la elaboración e implementación de las RIAS; se creó el grupo desarrollador de la ruta; se priorizaron y describieron las intervenciones en función del proceso continuo de atención en salud; se evaluó la práctica asistencial actual con grupos focales de pacientes y profesionales; se formularon los resultados esperados en el proceso de gestión y atención en salud (hitos), y se elaboró el diagrama de la ruta. Resultados: A partir de la Guía de práctica clínica se elaboraron 25 intervenciones individuales priorizadas y caracterizadas según el actor responsable, la población objetivo y el entorno. Para cada una de las intervenciones se presentan resultados esperados en salud, calidad de prestación de servicios, aspectos relacionados con la equidad, y la perspectiva de pacientes y actores involucrados con la atención. Se construyeron los indicadores para el seguimiento e implementación de la ruta. Conclusión: Se construyó la primera ruta integral de atención en salud del paciente con amputación de miembro inferior, de acuerdo con los lineamientos del manual del Ministerio de Salud y de la Protección Social.

-Palabras clave: adaptación protésica, amputación de miembro inferior, indicadores en salud, intervenciones individuales, ruta integral de atención en salud.

\section{Abstract}

Objective: Build an Integral Health Care Pathway for the care of patients with lower limb amputation due to traumatic, vascular or diabetes mellitus causes, in order to implement

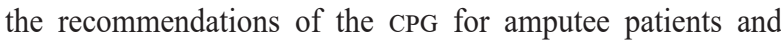
guarantee comprehensive health care for this population in Colombia. Methodology: This study is a strategy to improve health care. Carried out by a review of the Methodological Manual for the Development and Implementation of Comprehensive Health Care Pathway, then the development group was created. A process of prioritization and description of required individual interventions was developed based on health care. Evaluation of current care practice with focus groups, formulation of milestones and development of the intervention diagram. Results: 25 individual interventions were prioritized and characterized according to the responsible actor, target population and environment. Expected results in health, quality of service delivery, issues related to equity, as well as the perspective of patients and actors involved with care are shown. Indicators were built for monitoring and implementation of the pathway. Conclusion: With the previous results, the first Integral Health Care Pathway for the Lower Limb Amputee Patient was developed. It intends to guide the actors involved, when executing individual interventions for the diagnosis, treatment and rehabilitation, to impact outcomes in health and equity of this group.

-Keywords: Integral Health Care Pathway, lower limb amputation, individual interventions, health indicators, prosthetic adaptation.

\section{Resumo}

Objetivo: Construir uma Rota de Atenção Integral à Saúde do Paciente Amputado do Membro Inferior por causas traumáticas, vasculares ou diabetes mellitus, com a finalização de implementar as recomendações do GPC do paciente amputado e garantir a atenção integral na saúde desta población em Colômbia. Metodologia: Este estudo responde a uma estratégia para melhorar os cuidados de saúde. Foi realizada uma revisão do Manual Metodológico para o Desenvolvimento e Implementação de Rotas Integrais de Atenção à Saúde, criação do grupo de desenvolvimento da rota. Um processo de priorização e descrição das intervenções individuais necessárias foi desenvolvido com base na continuidade dos cuidados de saúde. Avaliação da prática assistencial atual com grupos focais, formulação de marcos e desenvolvimento do diagrama de intervenção. Resultados: 25 intervenções individuais foram priorizadas e caracterizadas de acordo com o ator responsável, população-alvo e ambiente. Determinação dos resultados esperados em saúde, qualidade da prestação de serviços, questões relacionadas a equidade, bem como a perspectiva de pacientes e atores envolvidos no atendimento. Foram construídos indicadores para o monitoramento e implementação da rota. Conclusão: Com os resultados anteriores, foi construída a primeira Rota de Atenção Integral à Saúde do paciente com amputação de membros inferiores por causas traumáticas e neurovasculares, com sua implementação, visando orientar os atores envolvidos na execução de intervenções individuais para a diagnóstico, tratamento e reabilitação, impactar os resultados em saúde e eqüidade dessa população.

-Palavras-chave: Rota Integral à Saúde, amputação de membros inferiores, intervenções individuais, indicadores de saúde, adaptação protética. 


\section{Introducción}

La National Health Interview Survey, en Estados Unidos, en 1996, reportó alrededor de 159000 amputaciones anuales de miembros inferiores [1]. En el año 2005, 1,6 millones de personas vivían con la pérdida de una extremidad, y se estima que sean más del doble en el año 2050 (3,6 millones) [2]. La diabetes mellitus y la enfermedad vascular periférica son las principales causas de amputación de miembro inferior [3]; estas enfermedades representan el $82 \%$ de los egresos hospitalarios por pérdida de una extremidad [4]. La diabetes mellitus, principal causa de amputaciones en el mundo, es también una de las principales causas de mortalidad; ocupa el sexto lugar, con una tasa de 28,98 por cada 100000 , de acuerdo con las estimaciones del "Estudio de carga global de la enfermedad", de 2017 [5]. La incidencia anual ajustada por edad, para las amputaciones no traumáticas de extremidades inferiores en personas diabéticas, varía de 2,1 a 13,7 por 1000 personas y puede ser 10 veces más frecuente que en la población general [6, p. 217;7].

A un taller de aparatos ortopédicos en Medellín, entre 2007 y 2016, de 3076 pacientes con amputación de miembro inferior, fueron por causas médicas el $54 \%$; de estas, por enfermedades neurovasculares, el $71 \%$. Por origen traumático asistieron el $46 \%$, y de estas últimas, por accidentes de tránsito, el $61 \%$ [8].

Según el Observatorio de Minas Antipersona (MAP) en Colombia, desde 1990 hasta enero 2020, se registraron 11811 víctimas de minas antipersonal y munición sin explosionar, de las cuales 111 se presentaron en el año 2019. Antioquia es el departamento con más víctimas civiles, seguido por Nariño y Meta [9]. Además, se calcula que el $70 \%$ de estas personas sufren amputación de una extremidad [10, p. 123].

Existen otras etiologías relacionadas con la amputación del miembro inferior, como el cáncer, la infección y los defectos congénitos de las extremidades inferiores [11].

El Grupo de Rehabilitación en Salud (GRES) de la Facultad de Medicina lideró, en la Universidad de Antioquia, la Guía de práctica clínica para el diagnóstico $y$ tratamiento preoperatorio, intraoperatorio y postoperatorio de la persona amputada, la prescripción de la prótesis y la rehabilitación integral (GPC), financiada por el Ministerio de Salud y Protección Social [12]. En esta GPC se buscó sistemáticamente la mejor evidencia disponible de la literatura médica y se desarrollaron recomendaciones para diagnóstico y tratamiento preoperatorio, intraoperatorio y postoperatorio para personas amputadas del miembro inferior [12].

Posteriormente, el GRES realizó una investigación para identificar barreras y facilitadores individuales y del sistema de salud que existen para el proceso de implementación de la GPC. Se reconocieron las perspec- tivas de los participantes: pacientes, trabajadores de la salud y personal administrativo de instituciones prestadoras de servicios en salud. Las barreras más relevantes encontradas fueron: la falta de formación académica en medicina basada en la evidencia y en GPC; respecto al funcionamiento del sistema de salud, la ausencia de redes integradas entre instituciones de diferentes niveles de complejidad; el gran número de procedimientos administrativos para los pacientes; la carencia de sistemas de información y la falta de difusión de las pautas para el cuidado de las personas con amputaciones [13].

Existe una legislación, como la Ley Estatutaria de Salud 1751 de 2015, cuyo objetivo es "garantizar el derecho a la salud, regularlo y establecer sus mecanismos de protección". Además, esta Ley establece que "el Estado adoptará políticas para asegurar la igualdad de trato y oportunidades en el acceso a las actividades de promoción, prevención, diagnóstico, tratamiento, rehabilitación y paliación para todas las personas" [14]. En el marco de esta ley estatutaria, la Ley 1955 de 2019 - por la cual se expide el Plan Nacional de Desarrollo 20182022, "Pacto por Colombia, pacto por la equidad"- define como objetivo "sentar las bases de legalidad, emprendimiento y equidad que permitan lograr la igualdad de oportunidades para todos los colombianos" [15]. Para lograrlo, se establecen, entre otros, el pacto por la inclusión de todas las personas con discapacidad y el pacto por una gestión pública efectiva [15].

La Resolución 489 [16] y la Ley 2626 de 2019 [17] definieron el modelo operacional de la "Política de atención integral en salud" y adoptaron el modelo de acción integral territorial (MAITE) [18]. Para la implementación del MAITE, se tienen en cuenta componentes operacionales, como la ruta integral de atención en salud (RIAS), entendida como "una herramienta que define a los agentes del Sistema de Salud (territorio, asegurador, prestador) y de otros sectores, las condiciones necesarias para asegurar la integralidad en la atención [...], a partir de las acciones orientadas a promover el bienestar y el desarrollo de los individuos en los entornos en los cuales se desarrolla, así como las intervenciones para la prevención, diagnóstico, tratamiento, rehabilitación de la discapacidad y paliación" [19]. Así mismo, el Ministerio de Salud y Protección Social (MSPs) diseñó el Manual metodológico para la elaboración e implementación de las RIAS, que pretende "brindar orientaciones técnicas a los integrantes del sistema de salud en el país para la elaboración, implementación, seguimiento y evaluación de las Rutas Integrales de Atención en Salud, como el elemento central para garantizar la atención integral en salud a la población colombiana, mediante la implementación del Modelo Integral de Atención en Salud en los territorios, atendiendo al proceso de adopción y adaptación de las RIAS de acuerdo con las particularidades territoriales y poblacionales" [19]. 
De esta manera, el objetivo de esta investigación fue la construcción de una RIAS para la atención del paciente amputado de miembro inferior por causas traumática, vascular o diabetes mellitus, con el fin de implementar las recomendaciones de la GPC del paciente amputado y garantizar la atención integral en salud de esta población en Colombia.

\section{Metodología}

Este fue un estudio descriptivo, de investigación científica, desarrollo tecnológico y transferencia del conocimiento, que responde a una estrategia de mejoramiento de la atención en salud.

De acuerdo con el manual metodológico desarrollado por el MSPS, la RIAS del paciente amputado es para eventos específicos. Su población objetivo son personas mayores de 16 años que van a ser o fueron amputadas del miembro inferior, cuya etiología haya sido médica (isquemia crítica de miembros inferiores, enfermedad arterial obstructiva crónica o pie diabético con clasificación de Wagner 3, 4 y 5) o secundaria a trauma civil o militar [12].

El lugar de aplicación principal de la RIAS del paciente amputado de miembro inferior es el ámbito asistencial y el propósito es organizar las acciones, a partir de recomendaciones basadas en la evidencia, para la atención en instituciones de mediana y alta complejidad del Sistema General de Seguridad Social en Salud, y en las instituciones prestadoras de servicios (IPS) de salud públicas y privadas $[19,20]$. De esta manera, su implementación implica la participación coordinada de diferentes actores del sistema de salud, las organizaciones sociales, públicas y privadas, y la comunidad.

Para esta investigación, se realizaron los siguientes pasos:

1. Revisión del Manual metodológico para la elaboración e implementación de las RIAS propuesto por el MSPS [19].

2. Creación del grupo desarrollador de la RIAS, conformado por dos estudiantes de posgrado en Medicina Física y Rehabilitación, un gerente en sistemas de información en salud, tres médicos fisiatras epidemiólogos, una enfermera doctora en Ciencias de la Salud Pública, y asesoría de dos profesionales doctorados en Políticas de Salud.

3. Identificación y selección de los grupos de riesgo priorizados por el MSPS, a partir de dicho Manual. En este proceso se describieron el grupo de riesgo y los eventos priorizados, la caracterización epidemiológica, la distribución geográfica, las metas y estrategias establecidas en el Plan Decenal de Salud Pública de Colombia, los compromisos nacionales e internacionales relacionados con el grupo de riesgo y el análisis de la situación en salud territorial (ASIS) [5]. La población con amputaciones de miembros inferiores se clasifica dentro del riesgo de alteraciones cardio-cerebro-vascular-metabólicas manifiestas y población con riesgo o sujeto de agresiones, accidentes y traumas.

4. Se definieron las intervenciones individuales requeridas, en función del continuo de la atención en salud del paciente amputado o con extremidad en riesgo. Estas intervenciones - pruebas diagnósticas, tratamientos médicos, quirúrgicos y de rehabilitaciónfueron tomadas de las recomendaciones basadas en la evidencia de la GPC [12], en la que participó un grupo interdisciplinario de cirujanos, fisiatras, terapia física, ocupacional, profesionales certificados en la prescripción de prótesis, representantes de universidades y de asociaciones científicas, provenientes de varias ciudades de Colombia.

Los ocho coinvestigadores evaluaron 43 intervenciones recomendadas en la GPC, según su relevancia y factibilidad; para cada criterio se calificaron de manera dicotómica (Sí o No). Se definieron como intervenciones priorizadas "aceptadas" las que cumplieran con los dos criterios. De acuerdo con esta calificación y sin presentarse desacuerdos entre los participantes, se priorizaron 19 recomendaciones; además, se tuvieron en cuenta 13 priorizadas en la actualización de la GPC del 2019. Siete de estas coincidieron con las priorizadas por el grupo de coinvestigadores, quedando al final 25 intervenciones.

1. Las intervenciones priorizadas se describieron según el agente responsable en el sistema ("quién"), la población objetivo de la intervención, la tecnología usada ("cómo") y el nivel de complejidad donde se desarrolla ("dónde").

2. Se especificaron los resultados esperados en el proceso de atención en salud, la calidad de la prestación de servicios y la reducción de la inequidad en salud. Para la inequidad se tuvo en cuenta la herramienta PROGRESS-Plus, la cual permite identificar desigualdades, ayudando a garantizar que los factores de estratificación social se tengan en cuenta en la toma de conductas, así como la discriminación por edad y la discapacidad [21].

3. Otro aspecto importante en el desarrollo de la ruta fue el reconocimiento de buenas y malas prácticas asistenciales actuales y de las intervenciones priorizadas, para lo cual se planearon dos actividades:

7.1. Un grupo focal, con siete pacientes de diferentes características y perfiles de riesgo; algunos estaban en proceso de adquisición de la prótesis, quienes firmaron el consentimiento informado. Tres facilitadores guiaron las entrevistas semiestructuradas, que fueron audiograbadas.

7.2. Una entrevista a dos profesionales expertos con experiencia en la atención del paciente amputa- 
do de miembro inferior. La identificación de las buenas y las malas prácticas de las intervenciones priorizadas para la prescripción de la prótesis se hizo con base en la eficiencia, la efectividad y la seguridad de cada intervención, de acuerdo con lo documentado en la GPC. Cada uno de los participantes calificó las intervenciones con una escala tipo Likert, desde "1" completamente en desacuerdo, a "5", completamente de acuerdo. La calificación mayor de 4 se consideró una buena práctica asistencial; por debajo de 4 , mala práctica [19].

4. La RIAS se basó en la GPC desarrollada de acuerdo con lineamientos de la Guía metodológica para la elaboración de guías de práctica clínica con evaluación económica en el Sistema General de Seguridad Social en Salud colombiano del MSPs [22]. Fue publicada en 2015 y actualizada en 2019. Con base en ella, se describieron la calidad de la evidencia, el balance riesgo/beneficios, los costos y los aspectos de equidad de cada intervención priorizada.

5. Posteriormente, se formularon y caracterizaron los hitos de la atención en salud, entendidos como resultados trazadores, ${ }^{*}$ a partir de las intervenciones definidas, y teniendo en consideración los parámetros sugeridos por el Manual metodológico para la elaboración e implementación de las RIAS [19], determinando los indicadores que permitieran la medición y el seguimiento de cada hito.

6. Se describieron, para cada una de las intervenciones de la RIAS, posibles factores facilitadores y barreras para la implementación.

7. Por último, se procedió a la construcción de la matriz de intervenciones para sintetizar la información de la RIAS y a la elaboración del diagrama de intervenciones. Para el registro y el análisis de la información se utilizó el programa de Microsoft Office Excel ${ }^{\circledR} 2019$ (v19.0), licencia de la Universidad de Antioquia; los gráficos de la ruta fueron hechos en el programa de modelación Bizagi modeler 3.6.0.044 [23], de uso libre, recomendado por el Manual metodológico para la elaboración e implementación de las RIAS.

La socialización y la validación externa de la RIAS se hicieron en foro virtual, con la participación de 61 actores clave, entre ellos, funcionarios del MSPS, profesionales de diferentes entidades promotoras de salud, IPS, universidades, sociedades científicas y pacientes.

\section{Consideraciones éticas}

La investigación cumple con los parámetros de la Resolución 8430 de 1993, artículos 1 y 23, considerándose una investigación con riesgo mínimo [24].

Los participantes firmaron consentimiento de su participación, una vez fueron informados de sus objetivos y riesgos.

\section{Resultados}

Las 25 intervenciones individuales requeridas en función del continuo de la atención en salud del paciente amputado se distribuyeron así: 1 estaba relacionada con diagnóstico; 10, con técnica y decisión quirúrgica, y 14, con el proceso de rehabilitación y adaptación de la prótesis, las cuales se describieron definiendo quién, cómo y dónde se llevan a cabo (véase Anexo 1).

Los actores responsables de las recomendaciones quirúrgicas son médicos especialistas en ortopedia, cirugía vascular y cirugía plástica; de las recomendaciones en rehabilitación y prótesis, los médicos fisiatras, fisioterapeutas y terapeutas ocupacionales. En algunas recomendaciones se requiere la participación de equipos multidisciplinarios. Estas se deben desarrollar en entornos institucionales, en el ámbito asistencial hospitalario o ambulatorio, en un nivel de complejidad mediano o alto.

A las intervenciones quirúrgicas y de rehabilitación se les definieron los resultados esperados en salud, en la calidad de la prestación de servicios y en la disminución de inequidad en salud (véanse Tabla 1 y Anexo 2).

Los resultados en la evaluación de la práctica actual fueron: los pacientes concluyeron que en la atención hospitalaria experimentan dificultades, como falta de comunicación por parte de los profesionales, ausencia de acompañamiento por el área psicosocial y muchos de ellos no contaron con tratamiento interdisciplinario. En la atención ambulatoria, en el proceso de rehabilitación, prescripción y adaptación protésica, se destaca la dificultad en el acceso a la consulta de fisiatría por falta de oportunidad. Los pacientes no reciben educación sobre los cuidados del muñón y la prótesis. Un paciente dice:

La mayoría de la gente desconoce que el sistema de salud le debe dar una prótesis, inclusive si usted está afiliado por el régimen subsidiado, porque yo he tenido prótesis por el régimen subsidiado (Tomado de relatoría grupo focal desarrollado con pacientes).

El resultado trazador es aquel hito que es susceptible de ser verificado tanto por el acto médico, como por medio de actos administrativos o de facturación de los procedimientos o un conjunto de procedimientos médicos o quirúrgicos [19]. 
Tabla 1. Principales resultados esperados en salud, en la calidad de la prestación de servicios y reducción de la inequidad en salud

\begin{tabular}{|c|c|c|c|}
\hline \multirow[b]{2}{*}{ Intervenciones } & \multicolumn{3}{|c|}{ Resultados esperados } \\
\hline & En salud & $\begin{array}{c}\text { En la calidad de la prestación } \\
\text { de los servicios }\end{array}$ & $\begin{array}{l}\text { En reducción de la inequidad en } \\
\text { salud }\end{array}$ \\
\hline \multirow{8}{*}{ Rehabilitación } & $\begin{array}{l}\text { Mejoría en desenlaces en } \\
\text { el componente mental y } \\
\text { psicológico }\end{array}$ & \multirow[t]{8}{*}{$\begin{array}{l}\text { Se espera adaptación } \\
\text { protésica exitosa, de acuerdo } \\
\text { con las necesidades del } \\
\text { paciente, y con ello no } \\
\text { aumentar costos y disminuir el } \\
\text { número de nuevas consultas }\end{array}$} & $\begin{array}{l}\text { Aplicar todas las intervenciones } \\
\text { en la población objetivo, } \\
\text { independientemente de edad, sexo, } \\
\text { raza, religión y educación, lo que } \\
\text { favorece la equidad }\end{array}$ \\
\hline & $\begin{array}{l}\text { Mejoría en la baja de la } \\
\text { intensidad del dolor neuropático }\end{array}$ & & $\begin{array}{l}\text { Favorecer la disminución de la } \\
\text { inequidad en la ocupación, al reducir } \\
\text { el dolor, la infección y la tasa de } \\
\text { reamputación }\end{array}$ \\
\hline & $\begin{array}{l}\text { Mejoría en independencia en } \\
\text { actividades de la vida diaria } \\
\text { (AVD) }\end{array}$ & & $\begin{array}{l}\text { Al mejorar la adaptación protésica, } \\
\text { el paciente podrá reintegrarse } \\
\text { laboralmente y lograr una } \\
\text { independencia en AVD }\end{array}$ \\
\hline & $\begin{array}{l}\text { Mejoría en aspectos funcionales } \\
\text { (patrón de marcha) }\end{array}$ & & $\begin{array}{l}\text { Mejorar la percepción de la } \\
\text { apariencia con la prótesis y se } \\
\text { espera que reduzca la inequidad por } \\
\text { discriminación }\end{array}$ \\
\hline & $\begin{array}{l}\text { Disminución del tiempo para la } \\
\text { adaptación protésica definitiva }\end{array}$ & & \multirow{4}{*}{$\begin{array}{l}\text { Favorecer la equidad en el capital } \\
\text { social, ya que al disminuir el dolor, la } \\
\text { estancia hospitalaria y las reconsultas, } \\
\text { y al mejorar la condición física y la } \\
\text { adaptación protésica, el paciente } \\
\text { participará en la sociedad en forma } \\
\text { temprana }\end{array}$} \\
\hline & $\begin{array}{l}\text { Satisfacción cosmética con la } \\
\text { prótesis }\end{array}$ & & \\
\hline & $\begin{array}{l}\text { Facilidad en el retorno a su } \\
\text { ocupación }\end{array}$ & & \\
\hline & $\begin{array}{l}\text { Reintegro laboral (parcial o total) } \\
\text { y en la participación social }\end{array}$ & & \\
\hline Quirúrgicas & $\begin{array}{l}\text { Disminuir el riesgo de infección } \\
\text { del muñón, el dolor agudo y } \\
\text { crónico de este, y el riesgo } \\
\text { de reintervención; mejorar la } \\
\text { cicatrización }\end{array}$ & $\begin{array}{l}\text { Disminución en estancia } \\
\text { hospitalaria, reintervenciones, } \\
\text { infecciones y costos }\end{array}$ & $\begin{array}{l}\text { Disminuir la inequidad en las } \\
\text { relaciones dependientes del tiempo, } \\
\text { al reducir las infecciones y prevenir la } \\
\text { realización de una reamputación; de } \\
\text { esta manera, el paciente se podría } \\
\text { integrar de forma más temprana a la } \\
\text { sociedad }\end{array}$ \\
\hline
\end{tabular}

En el grupo de profesionales expertos, se concluyó que la mayoría de los pacientes se logran adaptar a la prótesis, pero que las técnicas quirúrgicas influyen en el logro de dicha adaptación; por ejemplo, refuerzan la idea de la importancia de la longitud del muñón, aunque no es el único factor, pues, en ocasiones, por preservarla, no se garantiza un buen cubrimiento del muñón por los tejidos blandos. Reconocen que es posible que se adapten pocas prótesis en el posoperatorio inmediato a pacientes no oncológicos, por el poco entrenamiento que existe para ello.

La valoración de las intervenciones priorizadas determinó 13 intervenciones como "potencialmente de buena práctica asistencial" y 12 de ellas como de "buena práctica asistencial”. Ninguna fue valorada como intervención de mala práctica asistencial.

En la Tabla 2 se presenta un resumen del análisis de la evidencia y la calidad de la evidencia, balance riesgo/beneficios, costos y equidad de las intervenciones. En todas las intervenciones, los beneficios superaron los riesgos; por ejemplo, el uso de la analgesia epidural y de los medicamentos orales en monoterapia representa un beneficio en el control del dolor agudo posoperatorio y dolor neuropático, comparado con no usarlos, a pesar del riesgo de presentar efectos adversos asociados a estos. 


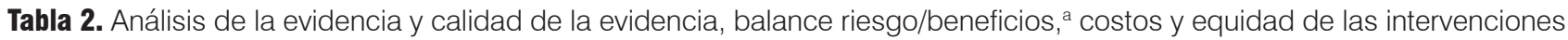

\begin{tabular}{|c|c|c|c|}
\hline Intervención & $\begin{array}{l}\text { Fuerza de la } \\
\text { recomendación }\end{array}$ & $\begin{array}{c}\text { Calidad de la } \\
\text { evidencia }\end{array}$ & Costos $^{b}$ \\
\hline $\begin{array}{l}\text { Pletismografía junto con presión arterial sistólica digital y presión arterial } \\
\text { sistólica en tobillo }\end{array}$ & Débil a favor & Muy baja & - \\
\hline $\begin{array}{l}\text { Procedimientos reconstructivos en muñón de amputación por debajo de la } \\
\text { rodilla }\end{array}$ & & & - \\
\hline Reconstrucción de la extremidad en pacientes con trauma grave & & Baja & $\begin{array}{l}\text { Costoefectiva a } \\
\text { largo plazo }\end{array}$ \\
\hline Amputación en dos tiempos & Fuerte a favor & Moderada & $\begin{array}{l}\text { Potencialmente } \\
\text { costoefectiva }\end{array}$ \\
\hline Analgesia epidural perioperatoria & Débil a favor & Muy baja & - \\
\hline Antibióticos profilácticos & Fuerte a favor & Baja & $\begin{array}{l}\text { Intervención } \\
\text { costoefectiva }\end{array}$ \\
\hline $\begin{array}{l}\text { Amputación del mediopié o retropié en pacientes con dos o más rayos } \\
\text { afectados }\end{array}$ & Débil a favor & & - \\
\hline Amputación transfemoral en lugar de desarticulación de la rodilla & & & - \\
\hline Miodesis en el muñón de amputación transfemoral & Fuerte a favor & Muy baja & $\begin{array}{l}\text { Potencialmente } \\
\text { costoefectiva }\end{array}$ \\
\hline Muñón óseo de por lo menos el 57 \% del fémur contralateral (transfemoral) & & Baja & - \\
\hline No usar sistemas cerrados de drenaje por succión & Débil en contra & Moderada & $\begin{array}{l}\text { Potencialmente } \\
\text { costoefectiva }\end{array}$ \\
\hline Prótesis inmediata postoperatoria & Débil a favor & Baja & - \\
\hline Adaptación de un pie tipo SACH en $\mathrm{K} 1^{\ddagger}$ & Fuerte a favor & & Intervención \\
\hline Adaptación de un pie articulado o uno de respuesta dinámica en K2/K3/K4 & Débil a favor & Muy baja & costoefectiva \\
\hline $\begin{array}{l}\text { Adaptación de una prótesis con cuenca de contacto total, amputación por } \\
\text { debajo de la rodilla }\end{array}$ & & Baja & \\
\hline $\begin{array}{l}\text { Prótesis con cuenca de contacto total, con encaje de interfaz en silicona, } \\
\text { copolímero o poliuretano. Amputación por debajo de la rodilla }\end{array}$ & & & - \\
\hline $\begin{array}{l}\text { Amputación transfemoral, rodilla monocéntrica con bloqueo manual o con } \\
\text { freno de carga }(\mathrm{K} 1) \text { y rodilla monocéntrica o policéntrica de control de } \\
\text { fluidos (K2, K3 y K4) }\end{array}$ & Fuerte a favor & & - \\
\hline $\begin{array}{l}\text { Rodilla policéntrica mecánica para desarticulado de rodilla }(K 1) \text { y policéntrica } \\
\text { de control de fluidos para desarticulación de rodilla (K2, K3 y K4) }\end{array}$ & Débil a favor & & - \\
\hline $\begin{array}{l}\text { Amputación por encima de la rodilla, y en niveles moderados o altos de } \\
\text { actividad, hacer adaptación de alguna de las variantes de cuenca de } \\
\text { contenimiento isquiático, y en niveles bajos de actividad, cuenca de tipo } \\
\text { cuadrilateral }\end{array}$ & Fuerte a favor & Muy baja & - \\
\hline $\begin{array}{l}\text { Adaptación individualizada de sistema de suspensión en amputación por } \\
\text { encima de la rodilla }\end{array}$ & & Baja & - \\
\hline $\begin{array}{l}\text { Tratamiento farmacológico para el dolor neuropático. Primera línea } \\
\text { pregabalina, seguida de gabapentina, amitriptilina y duloxetina como } \\
\text { monoterapia }\end{array}$ & & & $\begin{array}{l}\text { Intervención } \\
\text { costoefectiva }\end{array}$ \\
\hline $\begin{array}{l}\text { Programa de rehabilitación física (fuerza muscular, movilidad articular, } \\
\text { equilibrio, marcha) }\end{array}$ & & Muy baja & $\begin{array}{l}\text { Potencialmente } \\
\text { costoefectiva }\end{array}$ \\
\hline Rehabilitación ocupacional y las adaptaciones ergonómicas & & & - \\
\hline $\begin{array}{l}\text { Rehabilitación integral: cardiopulmonar, musculoesquelético, psicosocial, } \\
\text { actividades de la vida diaria y para el trabajo }\end{array}$ & & & - \\
\hline Escala de Houghton para evaluar la adaptación protésica & Débil a favor & Baja & - \\
\hline
\end{tabular}

${ }^{a}$ Balance riesgo / beneficios de la intervención: en todas las intervenciones los beneficios superan los riesgos [12].

${ }^{\mathrm{b}}$ El guion indica que no se hicieron análisis en estas recomendaciones.

${ }^{\mathrm{c}}$ Clasificación K de movilidad esperada propuesta por Medicare, de Estados Unidos. 
En algunas prescripciones protésicas, los beneficios en los resultados funcionales superan los inconvenientes, como la transpiración, el mal olor y el requerimiento de mayor habilidad por parte del paciente para usarla. Adicionalmente, las intervenciones quirúrgicas comprenden mayores beneficios, especialmente funcionales, y relacionados con menos complicaciones, comparado con los riesgos quirúrgicos inherentes.

Seis de las recomendaciones cuentan con análisis de costoefectividad, como, por ejemplo, la reconstrucción de la extremidad en pacientes con trauma grave. La fuerza de la recomendación fue fuerte a favor en 12 de ellas, débil a favor en otras 12 y débil en contra en 1 recomendación; la calidad de la evidencia está entre muy baja y moderada.

En el aspecto de equidad, todas las intervenciones están incluidas en el plan obligatorio de salud y su aplicación está sustentada por la Ley Estatutaria 1751 de
2015, por la cual se define el derecho fundamental a la salud en Colombia [14] (véase Tabla 2).

A partir de las recomendaciones definidas y agrupadas en las diferentes categorías, se formularon y caracterizaron los resultados que se esperan en el proceso de gestión y atención del paciente amputado, con el fin de garantizar su integralidad. Dichos resultados se determinaron como hitos de la atención y fueron: reamputación, reintervenciones por procesos infecciosos, adaptación protésica, reintegro laboral e independencia en actividades de la vida diaria. Se formularon los hitos o desenlaces, las intervenciones (recomendaciones) relacionadas con cada uno de ellos, el tipo y la fórmula de cálculo de los indicadores, y las fuentes de información. Para su formulación se tuvieron en cuenta la facilidad para ser medidos por medio de indicadores y las fuentes disponibles en los sistemas de información, como la historia clínica (véase Tabla 3 ).

Tabla 3. Caracterización, identificación de hitos de la atención

\begin{tabular}{|c|c|c|c|c|c|}
\hline \multirow{2}{*}{ Hito o desenlace } & \multirow{2}{*}{ Intervención } & \multirow{2}{*}{\multicolumn{2}{|c|}{$\begin{array}{l}\text { Tipo de indicador / Fórmula de } \\
\text { cálculo }\end{array}$}} & \multicolumn{2}{|c|}{ Fuente de información } \\
\hline & & & & Numerador & Denominador \\
\hline \multirow{6}{*}{$\begin{array}{l}\text { 1. Reamputación: } \\
\text { disminuir } \\
\text { reamputación }\end{array}$} & Intervenciones quirúrgicas: & \multicolumn{2}{|c|}{$\begin{array}{l}\text { Proporción de pacientes } \\
\text { reamputados }\end{array}$} & $\begin{array}{l}\text { Historias } \\
\text { clínicas }\end{array}$ & $\begin{array}{l}\text { Códigos } \\
\text { cups de }\end{array}$ \\
\hline & Reconstrucción del muñón & Tipo & Resultado & (reamputación & intervenciones: \\
\hline & debajo de la rodilla. & Frecuencia & Anual & & 8410,841001 \\
\hline & $\begin{array}{l}\text { Reconstrucción del miembro } \\
\text { inferior en trauma grave. }\end{array}$ & Meta & Disminuir reamputación & $\begin{array}{l}\text { reintervención) } \\
\text { Códigos cuPs: }{ }^{\dagger}\end{array}$ & $\begin{array}{l}-841003 \\
8412,841201\end{array}$ \\
\hline & Amputación en 2 tiempos. & Fór & $\begin{array}{l}<10 \% \text { año } \\
\text { Número de pacientes }\end{array}$ & $843,8436-$ & - 841202, \\
\hline & $\begin{array}{l}\text { Antibióticos profilácticos. } \\
\text { Amputación del mediopié } \\
\text { o retropie en dos o más }\end{array}$ & cálculo & $\begin{array}{l}\text { reamputados / Total } \\
\text { pacientes amputados }\end{array}$ & 8439. & $\begin{array}{l}841203, \\
841500,8413- \\
8418\end{array}$ \\
\hline \multirow{5}{*}{$\begin{array}{l}\text { 2. Reintervenciones } \\
\text { por procesos } \\
\text { infecciosos: } \\
\text { disminuir } \\
\text { reintervenciones }\end{array}$} & $\begin{array}{l}\text { rayos afectados por causas } \\
\text { isquémicas o por diabetes }\end{array}$ & \multicolumn{2}{|c|}{$\begin{array}{l}\text { Proporción de pacientes } \\
\text { reintervenidos en proceso infeccioso }\end{array}$} & & \\
\hline & Amputación transfemoral & Tipo & Resultado & & \\
\hline & No usar sistemas cerrados de & Frecuencia & Anual & & \\
\hline & $\begin{array}{l}\text { drenaje. } \\
\text { Intervenciones diagnósticas: } \\
\text { Pletismografía / presión arterial } \\
\text { sistólica diaital tobillo }\end{array}$ & Meta & $\begin{array}{l}\text { Disminuir } \\
\text { reintervenciones en el } \\
30 \%\end{array}$ & & \\
\hline & & $\begin{array}{l}\text { Fórmula } \\
\text { cálculo }\end{array}$ & $\begin{array}{l}\text { Número de pacientes } \\
\text { reintervenidos / Total } \\
\text { pacientes amputados }\end{array}$ & & \\
\hline
\end{tabular}




\begin{tabular}{|c|c|c|c|c|c|}
\hline \multirow{2}{*}{ Hito o desenlace } & \multirow{2}{*}{ Intervención } & \multirow{2}{*}{\multicolumn{2}{|c|}{$\begin{array}{l}\text { Tipo de indicador / Fórmula de } \\
\text { cálculo }\end{array}$}} & \multicolumn{2}{|c|}{ Fuente de información } \\
\hline & & & & Numerador & Denominador \\
\hline $\begin{array}{l}\text { 3. Adaptación } \\
\text { protésica: pacientes } \\
\text { adaptados a } \\
\text { prótesis medidas } \\
\text { con la escala de } \\
\text { Houghton }\end{array}$ & $\begin{array}{l}\text { Intervenciones protésicas: } \\
\text { Prótesis postoperatoria } \\
\text { inmediata. } \\
\text { Prescripción protésica: según } \\
\text { funcionalidad, nivel amputación, } \\
\text { condiciones geográficas y } \\
\text { ambientales. (Pie protésico, } \\
\text { rodilla protésica y cuenca, } \\
\text { interfaz y sistema suspensión) } \\
\text { Intervenciones en rehabilitación: } \\
\text { Tratamiento farmacológico para } \\
\text { el dolor neuropático } \\
\text { Programa de rehabilitación } \\
\text { física } \\
\text { Rehabilitación ocupacional y } \\
\text { ergonómica } \\
\text { Rehabilitación integral } \\
\text { Utiliza escala de Houghton } \\
\text { Intervención quirúrgica: } \\
\text { miodesis transfemoral y muñón } \\
\text { óseo de por lo menos el } 57 \text { \% } \\
\text { del fémur contralateral }\end{array}$ & $\begin{array}{l}\text { Proporción } \\
\text { a la prótesis } \\
\text { Tipo } \\
\text { Frecuencia } \\
\text { Meta } \\
\text { Fórmula: }\end{array}$ & $\begin{array}{l}\text { de pacientes adaptados } \\
\text { Resultado } \\
\text { Anual } \\
\text { Houghton > } 9 \\
\text { Número de paciente } \\
\text { adaptado a prótesis } \\
\text { / Total paciente con } \\
\text { prótesis }\end{array}$ & $\begin{array}{l}\text { Historia clínica } \\
\text { con escala de } \\
\text { Houghton }>9 \\
{[25]}\end{array}$ & $\begin{array}{l}\text { Códigos de } \\
\text { identificación } \\
\text { del dispositivo } \\
\text { según historia } \\
\text { clínica }\end{array}$ \\
\hline $\begin{array}{l}\text { 4. Reintegro laboral: } \\
\text { pacientes que } \\
\text { logran reintegro } \\
\text { laboral (parcial o } \\
\text { total) } \\
(<62 \text { años } \\
\text { hombres, }<57 \text { años } \\
\text { mujeres)* }[26]\end{array}$ & $\begin{array}{l}\text { Intervenciones en rehabilitación: } \\
\text { Tratamiento farmacológico para } \\
\text { el dolor neuropático } \\
\text { Programa de rehabilitación } \\
\text { física } \\
\text { Rehabilitación ocupacional y } \\
\text { ergonómica } \\
\text { Rehabilitación integral }\end{array}$ & $\begin{array}{l}\text { Proporción } \\
\text { reintegran } \\
\text { Tipo } \\
\text { Frecuencia } \\
\text { Meta } \\
\text { Fórmula }\end{array}$ & $\begin{array}{l}\text { de pacientes que se } \\
\text { Resultado } \\
\text { Anual } \\
\text { Reintegro } \\
\text { Número pacientes } \\
\text { reintegrados / Total } \\
\text { amputados }\end{array}$ & $\begin{array}{l}\text { Historia clínica } \\
\text { registro de } \\
\text { reintegro (Sí/ } \\
\text { No) }\end{array}$ & $\begin{array}{l}\text { Códigos } \\
\text { cups de } \\
\text { intervenciones: } \\
8410,841001 \\
\text { - 841003, } \\
8412,841201 \\
\text { - 841202, } \\
841203, \\
841500,8413 \text { - }\end{array}$ \\
\hline $\begin{array}{l}\text { 5. Independencia } \\
\text { en actividades } \\
\text { de la vida diaria } \\
\text { (AVD): pacientes } \\
\text { que logran } \\
\text { independencia en } \\
\text { AVD: subescala de } \\
\text { independencia en } \\
\text { AVD del WHODAS } \|^{\ddagger} \\
{[27,28]}\end{array}$ & Utiliza escala de Houghton & $\begin{array}{l}\text { Proporción } \\
\text { independen } \\
\text { Tipo } \\
\text { Frecuencia } \\
\text { Meta } \\
\text { Fórmula }\end{array}$ & $\begin{array}{l}\text { de pacientes con } \\
\text { cia en AVD } \\
\qquad \begin{array}{l}\text { Resultado } \\
\text { Anual } \\
80 / 100 \\
\text { Número } \\
\text { pacientes } \\
\text { con inde- } \\
\text { pendencia } \\
\text { en AvD / Total } \\
\text { amputados }\end{array}\end{array}$ & $\begin{array}{l}\text { Registro de } \\
\text { subescala de } \\
\text { independencia } \\
\text { en AVD del } \\
\text { WHODAS II }\end{array}$ & 8418 \\
\hline
\end{tabular}

*Edad de pensión en Colombia.

$\dagger$ Códigos únicos de procedimientos en salud.

† Cuestionario para la evaluación de la discapacidad, de la Organización Mundial de la Salud.

Las barreras y los facilitadores identificados con mayor frecuencia para las intervenciones individuales en el ámbito asistencial, tanto en el entorno hospitalario como ambulatorio, fueron las siguientes: falta de apoyos diagnósticos, de equipo e instrumental quirúrgico disponible; falta de recurso humano en los servicios según la complejidad; poca disponibilidad de servicios de rehabilitación en áreas lejanas; insuficientes IPS habili- tadas para llevar a cabo recomendaciones relacionadas con prótesis o rehabilitación; falta de adherencia a las GPC por parte de los profesionales de la salud, y falta de continuidad en el proceso de atención. Esta última barrera ha sido identificada por muchos actores como la más importante para obtener los logros planteados tanto en la GPC como en esta RIAS (véase Tabla 4). 
Tabla 4. Barreras y facilitadores para la implementación de intervenciones recomendadas en la RIAs del paciente amputado*

\begin{tabular}{|c|c|c|c|}
\hline \multicolumn{2}{|c|}{ Intervención } & Barrera & \multirow{2}{*}{\begin{tabular}{l}
\multicolumn{1}{c}{ Facilitador } \\
La recomendación es \\
expresada de manera \\
clara (ejecutabilidad)
\end{tabular}} \\
\hline Diagnóstico & $\begin{array}{l}\text { Pletismografía junto } \\
\text { con presión arterial } \\
\text { sistólica digital y } \\
\text { presión arterial } \\
\text { sistólica en tobillo }\end{array}$ & $\begin{array}{l}\text { No disponibilidad de apoyo diagnóstico en todos los } \\
\text { servicios. } \\
\text { No se cuenta con el perfil profesional para el desarrollo de la } \\
\text { intervención (Médico especialista en cirugía vascular) }\end{array}$ & \\
\hline \multirow[t]{4}{*}{$\begin{array}{l}\text { Intervención } \\
\text { quirúrgica }\end{array}$} & $\begin{array}{l}\text { Reconstruir en trauma } \\
\text { grave }\end{array}$ & $\begin{array}{l}\text { Poca disponibilidad: } \\
\text { Recurso humano (interdisciplinario). } \\
\text { Equipo e instrumental quirúrgico disponible. } \\
\text { Requiere periodos prolongados de tratamiento y } \\
\text { rehabilitación }\end{array}$ & $\begin{array}{l}\text { La recomendación } \\
\text { describe cómo } \\
\text { debe llevarse a } \\
\text { cabo la misma } \\
\text { (ejecutabilidad) }\end{array}$ \\
\hline & $\begin{array}{l}\text { Amputación en dos } \\
\text { tiempos }\end{array}$ & $\begin{array}{l}\text { El sistema de información actual no siempre permite } \\
\text { conocer las técnicas quirúrgicas aplicadas a cada paciente } \\
\text { (mensurabilidad) }\end{array}$ & \multirow{2}{*}{$\begin{array}{l}\text { Se describen las } \\
\text { condiciones que } \\
\text { deben cumplirse } \\
\text { para aplicar la } \\
\text { recomendación } \\
\text { (decidibilidad) }\end{array}$} \\
\hline & $\begin{array}{l}\text { Amputación del } \\
\text { mediopié o retropié en } \\
\text { pacientes con } 2 \text { o más } \\
\text { rayos afectados }\end{array}$ & $\begin{array}{l}\text { Falta de aceptación de la intervención por parte del paciente } \\
\text { o familiares }\end{array}$ & \\
\hline & $\begin{array}{l}\text { Miodesis en el muñón } \\
\text { de amputación } \\
\text { transfemoral }\end{array}$ & $\begin{array}{l}\text { El desconocimiento actual de algunos profesionales sobre la } \\
\text { medicina basada en la evidencia }\end{array}$ & $\begin{array}{l}\text { La recomendación } \\
\text { describe cómo debe } \\
\text { llevarse a cabo la } \\
\text { misma }\end{array}$ \\
\hline \multirow[t]{2}{*}{$\begin{array}{l}\text { Intervención en } \\
\text { rehabilitación }\end{array}$} & $\begin{array}{l}\text { Prótesis inmediata } \\
\text { postoperatoria }\end{array}$ & $\begin{array}{l}\text { No hay disponibilidad de insumos ni del perfil profesional } \\
\text { para el desarrollo de la intervención (protesista) en entorno } \\
\text { hospitalario inmediato }\end{array}$ & $\begin{array}{l}\text { La recomendación } \\
\text { describe cómo } \\
\text { debe llevarse a } \\
\text { cabo la misma } \\
\text { (ejecutabilidad) }\end{array}$ \\
\hline & $\begin{array}{l}\text { Programa de } \\
\text { rehabilitación física }\end{array}$ & $\begin{array}{l}\text { No disponibilidad de servicios de rehabilitación en áreas } \\
\text { retiradas. } \\
\text { No agilidad de los procesos administrativos. } \\
\text { Falta de continuidad en el proceso de atención. } \\
\text { Desconocimiento del equipo de salud sobre la estrategia. } \\
\text { Desconocimiento de los pacientes acerca de sus derechos } \\
\text { para la atención en rehabilitación }\end{array}$ & \multirow[t]{2}{*}{$\begin{array}{l}\text { La justificación de la } \\
\text { recomendación es } \\
\text { explícita }\end{array}$} \\
\hline Prótesis & Prescripción & $\begin{array}{l}\text { No hay suficientes ips ni talleres de aparatos ortopédicos } \\
\text { habilitados para probar o llevar a cabo las recomendaciones } \\
\text { relacionadas con prótesis o rehabilitación (efecto sobre el } \\
\text { proceso de atención). } \\
\text { La agilidad actual de los procesos de autorización y trámites } \\
\text { administrativos de los servicios de salud retrasa la ejecución } \\
\text { de esta intervención }\end{array}$ & \\
\hline
\end{tabular}

IPS: instituciones prestadoras de salud.

* Las barreras y facilitadores fueron elaborados basados en las recomendaciones de la Guía de práctica clínica para el diagnóstico y tratamiento preoperatorio, intraoperatorio y posoperatorio de la persona amputada, la prescripción de la prótesis y la rehabilitación integral [12] y la reunión de expertos de las RIAS.

Los facilitadores identificados fueron: La claridad con la que están expresadas las recomendaciones, la descripción detallada de las condiciones para su aplicación, la mayoría de las intervenciones se llevan a cabo en la práctica actual, y los profesionales están familiarizados con ellas y reconocen su efectividad.
En la Figura 1 se presenta el esquema de la RIAS del paciente amputado de miembro inferior. Este fue socializado en el proceso de validación externa realizado en el foro virtual.

En el foro deliberativo [29], los criterios de validez de la RIAS fueron evaluados favorablemente como cum- 


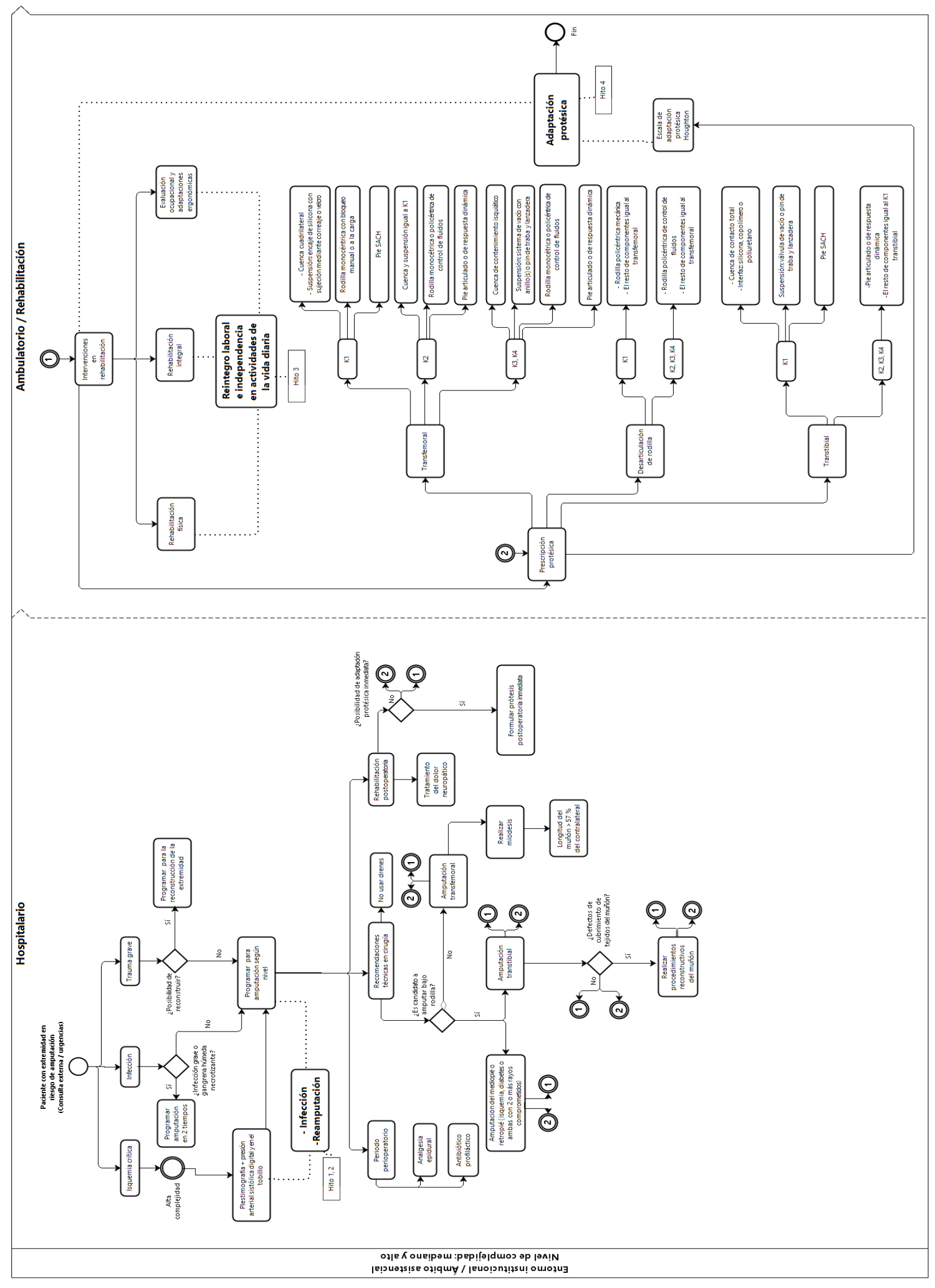


plidos, se identificaron oportunidades para su implementación y las conclusiones más importantes fueron:

1. Se deben fortalecer los procesos de educación tanto en los programas de pregrado y posgrado de las diferentes áreas implicadas en la atención del paciente amputado como los programas de educación continua, que les permitan a los profesionales tomar las mejores decisiones informadas.

2. La ley de interoperabilidad de historia clínica (Ley 2015 de 2020 [30]) es una oportunidad determinante para la mejora de los procesos de atención integral del paciente amputado; por lo tanto, los diferentes actores deben velar por su implementación.

3. Deben existir redes de atención integradas que garanticen la continuidad de la atención al paciente amputado y en riesgo de amputación; para esto, es fundamental la creación de un escenario ideal, donde los sistemas de información estén articulados y disponibles para la óptima atención del paciente.

4. Las rutas contemplan los diferentes actores responsables para cada paso de la atención y los hitos son verificables y rastreables en el proceso de atención, $\mathrm{y}$ repercuten en la retroalimentación del sistema de atención.

5. Los aseguradores deben reconocer su responsabilidad en la atención, rehabilitación y gestión del riesgo de su población asegurada.

6. Todos los actores deben comprometerse con la implementación de esta RIAS, para lograr una atención integral y de calidad al paciente amputado.

\section{Discusión}

La elaboración de esta RIAS para las personas amputadas estuvo enmarcada dentro de la Ley 1753 de 2015, donde se desarrolla el Plan Nacional de Desarrollo 2014-2018 [31]; la "Política de atención integral en salud", en la Ley 1751 de 2015 [14] del MSPS, y la Ley 1955 de 2019 [15]. El objetivo de esta RIAS, así como las demás que se hagan en Colombia, es "garantizar la atención integral en salud a las personas, familias y comunidades, a partir de intervenciones de valoración integral de la salud, detección temprana, protección específica, diagnóstico, tratamiento, rehabilitación, paliación y educación para la salud" [12].

Este trabajo se fundamentó en la GPC para el diagnóstico y el tratamiento del paciente con amputación de miembro inferior [12]. Las guías de práctica clínica se consideran herramientas importantes para mejorar la calidad, la seguridad del paciente y los costos de la atención; sin embargo, se han identificado diferentes dificultades para lograr la adherencia a las recomendaciones de las GPC. En una metarrevisión sistemática publicada en el 2020, que buscó explorar las barreras y los facilita- dores para la implementación de GPC en diferentes países, se encontró que las barreras más frecuentes fueron: la falta de claridad y de credibilidad en la evidencia de la GPC; la falta de conocimiento sobre esta; la falta de educación del personal en temas como la medicina basada en la evidencia; la ausencia de un líder del proceso de implementación dentro de las organizaciones y las creencias socioculturales de los pacientes [32].

Se ha reportado que la adherencia a las GPC es muy variable; puede estar entre el 30 y el $68 \%$ en países árabes; en Holanda se ha encontrado un promedio de adherencia del $61 \%$, y se encuentran reportes de adherencia del $35 \%$, especialmente en casos en los que las GPC no están basadas en evidencia científica, con recomendaciones vagas, complejas y controversiales [33,34]. En otra revisión sistemática realizada en Holanda, publicada en 2017, con 35 artículos incluidos, se encontró que los factores que más influían en la implementación son los tecnológicos, la creencia de que el uso de GPC obstaculiza la relación médico-paciente y los médicos consideraban que no necesitaban apoyo para la toma de decisiones. Entre los facilitadores más importantes fueron un proceso de formación práctico y personalizado, y la relevancia y facilidad de acceso a las recomendaciones [35].

En el año 2016, el Comité de Rehabilitación de Antioquia, en coordinación con el MSPS, hicieron una RIAS: "Ruta integral de atención en salud y de rehabilitación funcional para víctimas de MAP/MUSE". Esta ruta, a diferencia de la RIAS propuesta en este estudio, fue una guía para servidores públicos y personal del área de la salud, sobre las acciones que se deben hacer para activar la ruta de atención hasta el desarrollo de procesos de inclusión. Sus aspectos estratégicos fueron enfocados en la gestión y la autorización de servicios que requiere la víctima, como afiliación, financiamiento y trámites administrativos que tienen que hacer los prestadores de servicios de salud [36]. Existen otra RIAS enfocadas en acciones de gestión de salud pública para la promoción de la salud y la gestión de los principales riesgos de las personas, las familias y las comunidades. Se enfocan en actividades como la detección temprana, protección específica y educación en salud [37].

Esta RIAS del paciente amputado de miembro inferior por causas traumáticas, vasculares y diabetes orienta a los agentes del sistema de salud para la implementación de acciones en la atención integral de esta población. Su fortaleza se centra en que tuvo en cuenta las recomendaciones de la GPC del paciente amputado, financiada y adoptada por el MSPS. En esta ruta, el paciente se moviliza desde el ámbito asistencial hospitalario preoperatorio, operatorio y posoperatorio, hasta el ámbito ambulatorio para la rehabilitación y la adaptación a la prótesis, en niveles de complejidad mediano y alto. Sus resultados fueron encaminados a impactar positivamente la salud pública, pro- 
moviendo los derechos de uno de los grupos de personas con discapacidad, así como el acceso oportuno e integral al sistema de salud sin discriminación por ninguna condición individual, económica o social.

La implementación de esta RIAS y sus recomendaciones basadas en la evidencia mejorarán los desenlaces en salud, como: mejoría en la cicatrización; reducción del dolor agudo y crónico; aspectos funcionales relacionados con la marcha; independencia en AVD; reducción de complicaciones vinculadas con reintervenciones, estancia hospitalaria prolongada, infecciones y aumento de los costos. Esta implementación debe contribuir a la reducción de la inequidad, si se logra mejorar el reintegro laboral y la participación temprana en la sociedad. Lo anterior, sin aumentar los efectos adversos y con una adecuada relación costoefectividad.

La estrategia planteada para el seguimiento de la gestión y el cumplimiento de las intervenciones desarrolladas en la ruta fue la formulación de los siguientes hitos: reintervenciones por infecciones, reamputación, adaptación protésica, independencia en AVD y reintegro laboral. Estos deben ser medidos por medio de indicadores específicos de resultado; la fuente de información es la historia clínica. Se recomienda, para evaluar la adaptación protésica, la escala de Houghton [25], y para el logro de independencia en AVD, la subescala de independencia en AVD del WHODAS II [27,28]

La RIAS, si es implementada en el sector de la salud, puede ayudar a superar las barreras identificadas para la implementación de la GPC para las personas amputadas [13]. Por ello, y dada la importancia de los factores técnicos, organizacionales, así como los recursos humanos para implementar las GPC, la RIAS podría ser una estrategia importante para su implementación [35,38].

El análisis de la práctica actual de atención en salud actual y las limitaciones identificadas en la gestión de los procesos asistenciales dan cuenta de las barreras para el cumplimiento de las intervenciones en la población con amputación de miembro inferior. Por lo tanto, se hace necesario desarrollar estrategias encaminadas al mejoramiento del estado de salud, de la prestación de servicios con un enfoque apropiado de la gestión individual del riesgo y la elaboración de estrategias para la implementación de esta RIAS, particularmente en la atención integral del paciente y en la oportunidad para la prescripción de la prótesis y la rehabilitación.

Como fortalezas de este estudio, se destaca el empleo de la GPC para pacientes con amputación, avalada por el MSPs; la participación de un grupo interdisciplinario y representantes de asociaciones científicas, así como diferentes universidades en su elaboración; la opinión de expertos de diferentes áreas de conocimiento relacionadas con las fases del proceso de atención, así como la opinión de los pacientes. Las recomendaciones incluidas se pueden entender y la adherencia se puede medir, y brinda información sobre riesgos/beneficios de las intervenciones, lo cual permite aumentar la eficacia y la posibilidad de que se implementen [39].

Esta RIAS fue sometida a un proceso de verificación y validación interna, donde participaron médicos especialistas en medicina física y rehabilitación, epidemiólogos y profesionales en ciencias de la salud pública. Este proceso se evaluó y se ratificó en la validación externa realizada por medio de un foro deliberativo virtual, con la participación de los diferentes actores clave involucrados en la atención y la toma de decisiones del paciente amputado. Se resaltan como oportunidades para la implementación: los procesos educativos a profesionales y estudiantes, la ley de interoperabilidad de historia clínica, la atención integral y la participación de los aseguradores en el proceso de atención de la población amputada [29].

Una de las limitaciones encontradas fue la falta de datos epidemiológicos precisos registrados en los sistemas de información y en la literatura, que permitieran el desarrollo del Asis de la población objetivo. Otra de las limitaciones fue hacer un desarrollo de la gestión pública, pues este proceso recae en los entes territoriales; tampoco definimos un plan de implementación de esta RIAS, pero se tuvo el concurso de los diferentes funcionarios del MSPS, para definir los aspectos relacionados con la gobernanza, la monitorización, el proceso de descentralización de las acciones y las funciones, la rendición de cuentas y los mecanismos de incentivos.

Para futuras investigaciones, es importante realizar un seguimiento a la implementación de las intervenciones, para evaluar su adherencia y efectividad, además de utilizar los resultados de gestión e indicadores del proceso, propuestos en esta investigación, para mejorar los procesos de atención en salud de esta población. Estas mediciones estarán a cargo de las IPS o EPS que decidan implementar esta ruta. Además, los investigadores podrían evaluar la RIAS como una estrategia de implementación de las GPC para grupos poblacionales diferentes al expuesto en este trabajo.

\section{Agradecimientos}

Los autores agradecemos al grupo de rehabilitación en salud de la Universidad de Antioquia, a los profesionales y pacientes que participaron en el estudio.

\section{Conflicto de interés}

Los autores participaron en la actualización de la "Guía de práctica clínica para el diagnóstico y tratamiento preoperatorio, intraoperatorio y postoperatorio de la persona amputada, la prescripción de la prótesis y la rehabilitación integral 2019”. 
El Dr. Jesús Alberto Plata Contreras se desempeña como director técnico de la Corporación Mahavir Kmina.

\section{Financiación}

Esta investigación hace parte del Proyecto del Grupo de Rehabilitación en Salud de la Facultad de Medicina de la Universidad de Antioquia, titulado "Efectividad de una estrategia basada en Telesalud para mejorar la implementación de la "Guía de práctica clínica para el diagnóstico y tratamiento preoperatorio, intraoperatorio y postoperatorio de la persona amputada, la prescripción de la prótesis y la rehabilitación integral en instituciones de salud en Antioquia: un estudio de intervención aleatorizado por conglomerados de hospitales", el cual fue financiado por el Ministerio de Ciencia, Tecnología e Innovación (Código 111577757229) en la Convocatoria 777 del 2017.

\section{Declaración de responsabilidad}

Lo expresado en el documento es responsabilidad de los autores y no de la Universidad de Antioquia, ni de la Revista Facultad Nacional de Salud Pública.

\section{Declaración de autoría}

Laura Giraldo Castaño: participó en la concepción y el diseño del artículo, y en la adquisición, el análisis y la interpretación de los datos; llevó a cabo la revisión crítica del contenido intelectual; aprobó la versión final para ser publicada, y tiene la capacidad de responder por todos los aspectos del artículo, de cara a asegurar las cuestiones relacionadas con la exactitud o integridad de cualquier parte del trabajo.

Julieth Katerine Pinto Maquilón: participó en la concepción y el diseño del artículo, y en la adquisición, el análisis y la interpretación de los datos; llevó a cabo la revisión crítica del contenido intelectual; aprobó la versión final para ser publicada, y tiene la capacidad de responder por todos los aspectos del artículo, de cara a asegurar las cuestiones relacionadas con la exactitud o integridad de cualquier parte del trabajo.

Jesús Alberto Plata Contreras: participó en la concepción y el diseño del artículo, y en la adquisición, el análisis y la interpretación de los datos; llevó a cabo la revisión crítica del contenido intelectual; aprobó la versión final para ser publicada, y tiene la capacidad de responder por todos los aspectos del artículo, de cara a asegurar las cuestiones relacionadas con la exactitud o integridad de cualquier parte del trabajo.

Juan Carlos Velásquez Correa: participó en la concepción y el diseño del artículo, y en la adquisición, el análisis y la interpretación de los datos; llevó a cabo la revisión crítica del contenido intelectual; aprobó la versión final para ser publicada, y tiene la capacidad de responder por todos los aspectos del artículo, de cara a asegurar las cuestiones relacionadas con la exactitud o integridad de cualquier parte del trabajo.

María del Pilar Pastor: participó en la concepción y el diseño del artículo, y en la adquisición, el análisis y la interpretación de los datos; llevó a cabo la revisión crítica del contenido intelectual; aprobó la versión final para ser publicada, y tiene la capacidad de responder por todos los aspectos del artículo, de cara a asegurar las cuestiones relacionadas con la exactitud o integridad de cualquier parte del trabajo.

Ana María Posada Borrero: participó en la concepción y el diseño del artículo, y en la adquisición, el análisis y la interpretación de los datos; llevó a cabo la revisión crítica del contenido intelectual; aprobó la versión final para ser publicada, y tiene la capacidad de responder por todos los aspectos del artículo, de cara a asegurar las cuestiones relacionadas con la exactitud o integridad de cualquier parte del trabajo.

Daniel Felipe Patiño Lugo: participó en la concepción y el diseño del artículo, y en la adquisición, el análisis y la interpretación de los datos; llevó a cabo la revisión crítica del contenido intelectual; aprobó la versión final para ser publicada, y tiene la capacidad de responder por todos los aspectos del artículo, de cara a asegurar las cuestiones relacionadas con la exactitud o integridad de cualquier parte del trabajo.

Luz Helena Lugo Agudelo: participó en la concepción y el diseño del artículo, y en la adquisición, el análisis y la interpretación de los datos; llevó a cabo la revisión crítica del contenido intelectual; aprobó la versión final para ser publicada, y tiene la capacidad de responder por todos los aspectos del artículo, de cara a asegurar las cuestiones relacionadas con la exactitud o integridad de cualquier parte del trabajo.

\section{Referencias}

1. Owings MF, Kozak LJ. Ambulatory and inpatient procedures in the United States, 1996. Vital and health statistics [internet]. 1998 [citado 2020 oct. 5]; 13(139):1-127. Disponible en: https://www. cdc.gov/nchs/data/series/sr_13/sr13_139.pdf

2. Ziegler-Graham K, MacKenzie EJ, Ephraim PL, et al. Estimating the prevalence of limb loss in the United States: 2005 to 2050. Arch Phys Med Rehabil. 2008;89(3):422-9. https://doi. org/10.1016/j.apmr.2007.11.005

3. Braddom RL, Chan Leighton, Harrast MA. Chapter 13. Rehabilitation of people with lower limb amputation. En: D. Pepper, editor. Physical medicine and rehabilitation. 4th ed. Philadelphia: Saunders/Elsevier; 2011. pp. 277-316.

4. Dillingham T, Pezzin L, Mackenzie EJ. Limb amputation and limb deficiency: Epidemiology and recent trends in the United States. South Med J. 2002;95(8):875-83. Dor: https://doi. org/10.1097/00007611-200208000-00018 
5. Ministerio de Salud y Protección Social, Dirección de Epidemiología y Demografía. Análisis de situación de salud (ASIS)Colombia, 2018 Bogotá: Ministerio de Salud y Protección Social [internet]; 2018 [citado 2020 oct. 5]. Disponible en: https://www. minsalud.gov.co/sites/rid/Lists/BibliotecaDigital/RIDE/VS/ED/ PSP/asis-colombia-2018.pdf

6. Singh N, Armstrong DG, Lipsky BA. Preventing foot ulcers in patients with diabetes. JAMA. 2005;293(2):219-28. DoI: https://doi. org/10.1001/jama.293.2.217

7. Schoppen T, Boonstra A, Groothoff JW, et al. Employment status, job characteristics, and work-related health experience of people with a lower limb amputation in the Netherlands. Arch Phys Med Rehabil. 2001;82(2):239-45. DoI: https://doi.org/10.1053/ apmr.2001.18231

8. Pinto Maquilón JK, Giraldo Castaño L, et al. Evaluation related to functioning and prosthetic adaptation with low-cost exoskeletal prostheses in patients with lower limb amputations. Ann. Phys. Rehabil. Med.. 2018;61:e467-8. DoI: https://doi.org/10.1016/j.rehab.2018.05.1092

9. Oficina Alto Comisionado para la Paz. Víctimas de Minas Antipersonal y Municiones sin Explosionar [internet]. Informe a 31 de julio de 2021 [citado 2021 ago. 17]. Disponible en: http://www. accioncontraminas.gov.co/Estadisticas/Paginas/Estadisticas-deVictimas.aspx

10. Castaño-González A, Ceballos-González M, et al. Funcionamiento y estado de salud en una población de amputados de miembro inferior en Medellín, Colombia. Suplemento iatreia [internet]. 2016;29(4-S2):122-35. Disponible en: https://revistas.udea.edu. co/index.php/iatreia/article/view/325189

11. Godlwana L, Nadasan T, Puckree T. Global trends in incidence of lower limb amputation: A review of the literature. S Afr J Physiother. 2008;64(1):8-12. DOI: https://doi.org/10.4102/sajp. v64i1.93

12. Colombia, Ministerio de Salud y Protección Social. Guía de práctica clínica para el diagnóstico y tratamiento preoperatorio, intraoperatorio y postoperatorio de la persona amputada, la prescripción de la prótesis y la rehabilitación integral. Guía completa. Bogotá: Ministerio de Salud y Protección Social [internet]; 2015 [citado 2021 jul. 21 ]. Disponible en: http:// gpc.minsalud.gov.co/gpc_sites/Repositorio/Conv_637/GPC amputacion/GPC_AMP_completa.pdf

13. Patiño-Lugo D, Pastor Durango M, Lugo-Agudelo L, et al. Implementation of the clinical practice guideline for individuals with amputations in Colombia: A qualitative study on perceived barriers and facilitators. BMC Health Serv Res 2020;20(1):538. Dor: https://doi.org/10.1186/s12913-020-05406-z

14. Colombia, Congreso de la República. Ley Estatutaria 1751, por medio de la cual se regula el derecho fundamental a la salud y se dictan otras disposiciones (2015 feb. 16).

15. Colombia, Congreso de la República. Ley 1955, por la cual se expide el Plan Nacional de Desarrollo 2018-2022 "Pacto por Colombia, pacto por la equidad" (2019 may. 25).

16. Colombia, Ministerio de salud y protección social. Resolución 489, por la cual se modifica la Resolución 429 de 2016 (2019 feb. 2019).

17. Colombia, Ministerio de Salud y Protección Social. Resolución 2626, por la cual se modifica la Política de atención integral en salud -PAIs y se adopta el modelo de acción integral territorial -MAite (2019, sep. 27).

18. Colombia, Ministerio de Salud y Protección Social. Política de atención integral en salud. Bogotá [internet]; 2016 [citado 2020 oct. 5]. Disponible en: https://www.minsalud.gov.co/sites/rid/ Lists/BibliotecaDigital/RIDE/DE/modelo-pais-2016.pdf

19. Colombia, Ministerio de Salud y Protección Social. Manual metodológico para la elaboración e implementación de las RIAS. Bogotá [internet]; 2016 [citado 2020 oct. 5]. Disponible en: https:// www.minsalud.gov.co/sites/rid/Lists/BibliotecaDigital/RIDE/VS/ Manual-metodologico-rias.pdf

20. Colombia, Ministerio de Salud y Protección Social. Resolución 0518, por la cual se dictan disposiciones en relación con la gestión de la salud pública y se establecen directrices para la ejecución, seguimiento y evaluación del Plan de Salu Pública de Intervenciones Colectivas - PIC ( 2015 feb. 25).

21. O'Neill J, Tabish H, Welch V, et al. Applying an equity lens to interventions: Using PROGRESS ensures consideration of socially stratifying factors to illuminate inequities in health. J Clin Epidemiol. 2014;67(1):56-64. DOI: https://doi.org/10.1016/j.jclinepi.2013.08.005

22. Pulido C, De La Hoz AL, Mieth K, et al. Guía metodológica para la elaboración de guías de práctica clínica con evaluación económica en el Sistema General de Seguridad Social en Salud colombiano. Bogotá: Ministerio de Salud y Protección social [internet]; 2014 [citado 2021 jul. 19]. Disponible en: http://gpc. minsalud.gov.co/recursos/Documentos\%20compartidos/Guia Metodologica_Web.pdf

23. Bizagi - Intelligent Process Automation Leader [internet]. s. f. [citado 2020 mar. 7]. Disponible en: https://www.bizagi. com/?lang=en

24. Colombia, Ministerio de Salud y Protección Social. Resolución 8430 , por la cual se establecen las normas científicas, técnicas y administrativas para la investigación en salud (1993 oct. 4).

25. Houghton A, Allen A, et al. Rehabilitation after lower limb amputation: A comparative study of above-knee, through-knee and Gritti-Stokes amputations. Br J Surg. 1989;76(6):622-4. DoI: https://doi.org/10.1002/bjs.1800760633

26. Colombia, Congreso de la República. Ley 797, por la cual se reforman algunas disposiciones del Sistema General de Pensiones previsto en la Ley 100 de 1993 y se adoptan disposiciones sobre los Regímenes Pensionales Exceptuados y Especiales (2003 ene. 29).

27. Organización Mundial de la Salud. Medición de la salud y la discapacidad. Manual para el cuestionario de evaluación de la discapacidad de la OMS. WHODAs 2.0. Organización Mundial de la Salud [internet]; 2015 [citado 2020 oct. 5]. Disponible en: https://apps. who.int/iris/bitstream/handle/10665/170500/9874573309_spa. pdf? sequence $=1$ \&isAllowed $=\mathrm{y}$

28. Pösl M, Cieza A, Stucki G. Psychometric properties of the wHODASII in rehabilitation patients. Qual Life Res. 2007;16(9):152131. DoI: https://doi.org/10.1007/s11136-007-9259-4

29. UNED, Grupo de Rehabilitación en Salud (GRes). Verificación y validación externa de la ruta integral de atención en salud RIAS para amputaciones de miembro inferior por causas traumáticas y neurovasculares; 2020 [Documento interno de trabajo].

30. Colombia, Ministerio de Salud y Protección Social, Ley 2015, por medio del cual se crea la historia clínica electrónica interoperable y se dictan otras disposiciones (2020 ene. 31).

31. Colombia, Congreso de la República, Ley 1753, por la cual se expide el Plan Nacional de Desarrollo 2014-2018 "Todos por un nuevo país" (2015 jun. 9)

32. Correa V, Lugo-Agudelo L, Aguirre-Acevedo D, et al. Individual, health system, and contextual barriers and facilitators for the implementation of clinical practice guidelines: A systematic metareview. Health Res Policy Sys. 2020;18(1):74. Dor: https://doi. org/10.1186/s12961-020-00588-8 
34. Koornneef E, Robben P, et al. The development, implementation and evaluation of clinical practice guidelines in Gulf Cooperation Council (GCC) countries: A systematic review of literature. J Eval Clin Pract. 2015; 21(6):1006-13. DoI: https://doi.org/10.1111/ jep. 12337

34. Grol R, Dalhuijsen J, Thomas S, et al. Attributes of clinical guidelines that influence use of guidelines in general practice: Observational study. BMJ 1998;317:858-61. DOI: https://doi.org/10.1136/ bmj.317.7162.858

35. Kilsdonk E, Peute LW, Jaspers MWM. Factors influencing implementation success of guideline-based clinical decision support systems: A systematic review and gaps analysis. Int J Med Inform. 2017;98:56-64. DOI: https://doi.org/10.1016/j.ijmedinf.2016.12.001

36. Organización Internacional para las Migraciones (OIM), Ministerio de Salud y Protección Social, Agencia de los Estados Unidos para el Desarrollo Internacional (USAID), Dirección para la Acción Integral contra Minas Antipersonal E Comité de R. Ruta integral de atención en salud y de rehabilitación funcional para víctimas de MAP/MUSE. Bogotá [internet]; 2016 [citado 2021 jul. 21]. Disponible en: https:/www.minsalud.gov.co/sites/rid/Lists/ BibliotecaDigital/RIDE/DE/PS/ruta-integral-atencion-saludrehabilitacion-funcional-victimas-mapmuse.pdf
37. Colombia, Ministerio de Salud y Protección Social. Resolución 3280 , por medio de la cual se adoptan los lineamientos técnicos y operativos de la Ruta integral de atención para la promoción y mantenimiento de la salud y la Ruta integral de atención en salud para la población materno perinatal y se establecen las directrices para su operación (2018 ago. 2).

38. Prior M, Guerin M, Grimmer-Somers K. The effectiveness of clinical guideline implementation strategies -- a synthesis of systematic review findings. J Eval Clin Pract. 2008;14(5):888-97. DoI: https://doi.org/10.1111/j.1365-2753.2008.01014.x

39. Unverzagt S, Peinemann F, Oemler M, et al. Meta-regression analyses to explain statistical heterogeneity in a systematic review of strategies for guideline implementation in primary health care. PLoS ONE. 2014;9(10):e110619. https://doi.org/10.1371/journal. pone.0110619

40. Colombia, Ministerio de Salud y Protección Social. Resolución 00002003, por la cual se definen los procedimientos y condiciones de inscripción de los prestadores de servicios de salud y de habilitación de servicios de salud (2014 may. 28).

41. Colombia, Ministerio de Salud y Protección Social. Resolución 2968, por la cual se establecen los requisitos sanitarios que deben cumplir los establecimientos que elaboran y adaptan dispositivos médicos sobre medida de tecnología ortopédica externa ubicados en el territorio nacional (2015, ago. 14).

Esta obra se distribuye bajo una Licencia Creative Commons Atribución-NoComercial-CompartirIgual 4.0 Internacional

Más información: https://creativecommons.org/licenses/by-nc-sa/4.0/ 


\section{Anexos}

\section{Anexo 1. Identificación y descripción de las intervenciones.}

\begin{tabular}{|c|c|c|c|}
\hline Intervención & Quién & Cómo & Dónde [40] \\
\hline $\begin{array}{l}\text { Se sugiere realizar la pletismografía, junto } \\
\text { con la presión arterial sistólica digital y la } \\
\text { presión arterial sistólica en el tobillo, en caso } \\
\text { de no contar con la tensión transcutánea de } \\
\text { oxígeno, en pacientes mayores de } 16 \text { años } \\
\text { con alteraciones en la perfusión del pie que } \\
\text { van a ser sometidos a una amputación }\end{array}$ & $\begin{array}{l}\text { Médico especialista en cirugía } \\
\text { vascular }\end{array}$ & $\begin{array}{l}\text { Mayores de } 16 \text { años } \\
\text { con alteraciones en la } \\
\text { perfusión del pie que } \\
\text { van a ser sometidos a } \\
\text { una amputación }\end{array}$ & $\begin{array}{l}\text { Entorno institucional } \\
\text { Ámbito asistencial / } \\
\text { Hospitalario } \\
\text { Nivel de complejidad: } \\
\text { alto }\end{array}$ \\
\hline $\begin{array}{l}\text { Se sugiere la realización de procedimientos } \\
\text { reconstructivos de tejidos blandos, colgajos } \\
\text { libres o injertos, para el tratamiento de } \\
\text { defectos de cubrimiento de tejidos blandos } \\
\text { del muñón de amputación por debajo de } \\
\text { la rodilla, para conservar esta articulación y } \\
\text { mantener un nivel de amputación transtibial }\end{array}$ & $\begin{array}{l}\text { La realización de } \\
\text { procedimientos } \\
\text { reconstructivos de tejidos } \\
\text { blandos debe quedar a criterio } \\
\text { del cirujano. Es necesario que } \\
\text { se involucren en la toma de la } \\
\text { decisión otras especialidades } \\
\text { médico-quirúrgicas, como } \\
\text { ortopedia, cirugía plástica, } \\
\text { cirugía vascular, medicina } \\
\text { física y rehabilitación e } \\
\text { infectología }\end{array}$ & $\begin{array}{l}\text { Mayores de } 16 \text { años con } \\
\text { defectos de cubrimiento } \\
\text { de tejidos blandos del } \\
\text { muñón de amputación } \\
\text { (bajo rodilla) }\end{array}$ & $\begin{array}{l}\text { Entorno institucional } \\
\text { Ámbito asistencial } \\
\text { / Hospitalario - } \\
\text { Quirófano } \\
\text { Nivel de complejidad: } \\
\text { alto }\end{array}$ \\
\hline $\begin{array}{l}\text { Se sugiere realizar la reconstrucción de la } \\
\text { extremidad en pacientes con trauma grave } \\
\text { del miembro inferior, en lugar de amputación, } \\
\text { para mejorar la función y disminuir los costos }\end{array}$ & $\begin{array}{l}\text { Médico especialista en cirugía } \\
\text { plástica. } \\
\text { Otras especialidades médico } \\
\text { quirúrgicas como: ortopedia, } \\
\text { cirugía vascular, medicina } \\
\text { física y rehabilitación e } \\
\text { infectología }\end{array}$ & $\begin{array}{l}\text { Mayores de } 16 \text { años } \\
\text { con trauma grave del } \\
\text { miembro inferior }\end{array}$ & \\
\hline $\begin{array}{l}\text { Se recomienda la amputación en dos } \\
\text { tiempos, en lugar de la amputación en } \\
\text { un solo tiempo con cierre primario, para } \\
\text { disminuir el riesgo de infección del muñón, } \\
\text { mejorar la cicatrización y acortar el tiempo de } \\
\text { hospitalización }\end{array}$ & $\begin{array}{l}\text { Médico ortopedista, cirujano } \\
\text { vascular }\end{array}$ & $\begin{array}{l}\text { Mayores de } 16 \text { años } \\
\text { que requieren una } \\
\text { amputación del } \\
\text { miembro inferior, } \\
\text { secundaria a gangrena } \\
\text { húmeda necrotizante e } \\
\text { infecciones graves }\end{array}$ & $\begin{array}{l}\text { Entorno institucional } \\
\text { Ámbito asistencial } \\
\text { / Hospitalario - } \\
\text { Quirófano } \\
\text { Nivel de complejidad: }\end{array}$ \\
\hline $\begin{array}{l}\text { Se sugiere la analgesia epidural } \\
\text { perioperatoria, para disminuir el dolor agudo } \\
\text { del muñón y del miembro fantasma en el } \\
\text { periodo postoperatorio }\end{array}$ & Médico anestesiólogo & $\begin{array}{l}\text { Mayores de } 16 \text { años que } \\
\text { van a ser amputados de } \\
\text { miembros inferiores }\end{array}$ & \\
\hline $\begin{array}{l}\text { Se recomienda el uso de antibióticos } \\
\text { profilácticos, para la prevención de infección } \\
\text { del muñón }\end{array}$ & $\begin{array}{l}\text { Médico ortopedista o cirujano } \\
\text { vascular }\end{array}$ & $\begin{array}{l}\text { Mayores de } 16 \text { años } \\
\text { que requieran una } \\
\text { amputación del } \\
\text { miembro inferior, por } \\
\text { un periodo de tiempo } \\
\text { que no exceda las } 24 \\
\text { horas después de la } \\
\text { amputación. } \\
\text { Cefazolina intravenosa } \\
2 \text { gr, } 30 \text { a } 60 \text { minutos } \\
\text { antes de la incisión } \\
\text { quirúrgica, idealmente } \\
\text { una sola dosis, pero } \\
\text { nunca más de tres dosis } \\
\text { o } 24 \text { horas }\end{array}$ & $\begin{array}{l}\text { Entorno institucional } \\
\text { Ámbito asistencial / } \\
\text { Hospitalario } \\
\text { Nivel de complejidad: } \\
\text { mediano y alto }\end{array}$ \\
\hline
\end{tabular}




\section{Intervención}

Se sugiere la amputación del mediopié o retropié en pacientes que tengan afectados dos o más rayos por causas isquémicas o por diabetes, para disminuir la frecuencia de reintervención para subir el nivel de la amputación

Se sugiere la realización de una amputación transfemoral en lugar de desarticulación de la rodilla en los pacientes que requieren una amputación del miembro inferior y no son candidatos a una por debajo de la rodilla, para disminuir las infecciones del muñón, mejorar la satisfacción cosmética con la prótesis, disminuir el dolor en el muñón de amputación y la necesidad de reamputación

Se recomienda la realización de miodesis en el muñón de amputación, para permitir un adecuado balance de las fuerzas musculares en el muñón de amputación y mejorar su estabilidad dentro de la prótesis

Se recomienda, al realizar una amputación transfemoral, obtener un muñón óseo de por lo menos el $57 \%$ del fémur contralateral, para aumentar la velocidad y evitar la asimetría del patrón de la marcha secundario a alteraciones compensatorias en la inclinación del tronco o de la pelvis

No se sugiere, de forma rutinaria, el uso de sistemas cerrados de drenaje por succión después del cierre definitivo en pacientes que requieran una amputación del miembro inferior, para disminuir el riesgo de infección y necesidad de cirugías adicionales por hematomas o seromas

Tratamiento farmacológico, para el tratamiento del dolor neuropático.

Se recomienda, en primer lugar, la pregabalina, seguida de gabapentina, amitriptilina y duloxetina como monoterapia

Se sugiere el uso de una prótesis inmediata postoperatoria, para mejorar la remodelación del muñón, facilitar la adaptación protésica y el funcionamiento, y disminuir el tiempo para la adaptación protésica definitiva

\section{Quién}

Cómo

Mayores de 16 años que tengan afectados dos o más rayos por causas isquémicas o por diabetes, para disminuir la frecuencia de reintervención para subir el nivel de la amputación

Mayores de 16 años que requieren una amputación del miembro inferior y no son candidatos a una por debajo de la rodilla

Mayores de 16 años que requieran una amputación transfemoral por etiología traumática o vascular

Mayores de 16 años que requieren una amputación transfemoral por causas traumáticas, isquémicas o por diabetes

Mayores de 16 años que requieran una amputación del miembro inferior por causas traumáticas, isquémicas o por diabetes

Médico ortopedista, cirujano vascular, fisiatra

Mayores de 16 años, amputados por causas traumáticas, vasculares o por diabetes para reducir el dolor neuropático

Mayores de 16 años amputados de miembros inferiores por causas traumáticas y vasculares
Médico fisiatra
Dónde [40]

Entorno institucional

Ámbito asistencial

/ Hospitalario -

Quirófano

Nivel de complejidad: mediano y alto
Entorno institucional

Ámbito asistencial / Hospitalario o ambulatorio

Nivel de complejidad: mediano y alto

Entorno institucional

Ámbito asistencial / Hospitalario

Nivel de complejidad: mediano y alto 


\section{Intervención}

Se recomienda, en las personas con amputación por encima o debajo de la rodilla, la adaptación de un pie tipo $\mathrm{SACH}$, para mejorar la función de la marcha.

En pacientes adultos mayores con nivel funcional $\mathrm{K} 1$ * que hayan utilizado previamente un pie tipo SACH podría evaluarse la posibilidad de adaptación de un pie articulado

Se sugiere la adaptación de un pie articulado o uno de respuesta dinámica en personas con requerimientos mayores de actividad. Recomendado por un médico especialista con entrenamiento en el área de prótesis y que las condiciones sociales o ambientales lo posibiliten

Se sugiere la adaptación de una prótesis con cuenca de contacto total y funda de silicona en las personas con amputación por debajo de la rodilla, de acuerdo con las condiciones del muñón y las habilidades residuales del paciente para mejorar la función de la marcha

Se sugiere, en las personas con amputación por debajo de rodilla, una prótesis con cuenca de contacto total, con encaje de interface en silicona, copolímero o poliuretano. El uso de una válvula de vacío o un pin con traba y lanzadera debe ser individualizado teniendo en cuenta las condiciones físicas del paciente y el muñón, el nivel de actividad y su ámbito personal, social y ambiental

Se sugiere, en las personas con amputación por encima de la rodilla y un nivel esperado de actividad $\mathrm{K} 1$, * la adaptación de una rodilla monocéntrica con bloqueo manual o con freno de carga; en los K2, K3 y K4, * una monocéntrica o policéntrica de control de fluidos, para mejorar la función de la marcha en los primeros 12 meses luego de la amputación

Se sugiere, en las personas mayores de 16 años con desarticulación de rodilla y un nivel esperado de actividad K1, la adaptación de una rodilla policéntrica mecánica para desarticulado de rodilla; y en los K2, K3 y $\mathrm{K} 4$, una policéntrica de control de fluidos, para desarticulación de rodilla, para mejorar la función de la marcha en los primeros 12 meses luego de la amputación
Médico fisiatra con entrenamiento en el área de prótesis

Médico fisiatra

Médico fisiatra.

El sistema de control de fluidos (neumático o hidráulico) debe ser prescrito por un médico especialista con entrenamiento y experiencia en adaptación protésica y aprobado por un comité técnico-científico
Mayores de 16 años, con amputación por encima o debajo de la rodilla y un nivel esperado de actividad bajo (K1)

Cómo

Dónde [40]

Entorno institucional

Ámbito asistencial /

Ambulatorio

Nivel de complejidad: mediano y alto

Taller de prótesis inscrito en el Invima, según Resolución 2968 de 2015 [41]
Mayores de 16 años, con amputación de miembro inferior, con requerimientos mayores de actividad (K2/K3/K4) o que deban utilizar la prótesis en superficies irregulares o inclinadas

Mayores de 16 años con amputación por debajo de la rodilla, de acuerdo con las condiciones del muñón y las habilidades residuales del paciente

Mayores de 16 años con amputación por debajo de la rodilla
Mayores de 16 años con amputación por encima de la rodilla.

Nivel esperado de actividad K1 y K2, K3 y K4

Mayores de 16 años con desarticulación de rodilla.

Nivel esperado de actividad $\mathrm{K} 1$ y K2, K3

y K4 


\section{Intervención}

Se recomienda, en las personas con amputación por encima de la rodilla y niveles moderados o altos de actividad, la adaptación de alguna de las variantes de cuenca de contenimiento isquiático, para mejorar la función de la marcha. Se recomienda, en las personas con niveles bajos de actividad, la adaptación de una cuenca de tipo cuadrilateral

Se recomienda la adaptación individualizada de un sistema de suspensión acorde con las capacidades funcionales del paciente y la condición del muñón.

Para pacientes con niveles bajos de actividad (K1 y K2), se utilizan encajes de silicona con sujeción mediante correaje y velcro, o sujeción mediante cordón.

En pacientes muy activos (K3 y K4), se prefiere los sistemas de vacío, pues crean mejor confort, mejor sujeción y son más fáciles de adaptar por el paciente que los de pin y lanzadera

Se recomienda la implementación de un programa de rehabilitación física que incluya fuerza muscular, movilidad articular, equilibrio, marcha y reacondicionamiento físico, en pacientes con amputación de miembro inferior por causas traumáticas, vasculares o por diabetes, para mejorar la marcha, la movilidad y la adaptación protésica

Se recomienda la rehabilitación ocupacional y Médico fisiatra

las adaptaciones ergonómicas en pacientes a Terapeuta ocupacional quienes les fue amputado un miembro inferior, para mejorar el funcionamiento y facilitar el retorno al trabajo o a una ocupación

Se recomienda la implementación de un proceso de rehabilitación integral: cardiopulmonar, musculoesquelético, psicosocial, actividades de la vida diaria y para el trabajo, en pacientes con amputación de miembro inferior, de causas traumáticas, vasculares o por diabetes, para mejorar o mantener el funcionamiento, la independencia en las actividades de la vida diaria, el reintegro y la participación social
Médico fisiatra

Médico fisiatra, especialista con entrenamiento en el área de prótesis

Médico fisiatra

Terapeuta físico 


\begin{tabular}{|c|c|c|c|}
\hline Intervención & Quién & Cómo & Dónde [40] \\
\hline $\begin{array}{l}\text { Se sugiere la utilización de la Escala de } \\
\text { Houghton para evaluar la adaptación } \\
\text { protésica }\end{array}$ & $\begin{array}{l}\text { Médico fisiatra, terapeuta } \\
\text { físico }\end{array}$ & $\begin{array}{l}\text { Mayores de } 16 \text { años } \\
\text { a quienes les fue } \\
\text { amputado un miembro } \\
\text { inferior por causas } \\
\text { traumáticas, vasculares } \\
\text { o por diabetes }\end{array}$ & $\begin{array}{l}\text { Entorno institucional } \\
\text { Ámbito asistencial / } \\
\text { Ambulatorio } \\
\text { Nivel de complejidad: } \\
\text { mediano y alto }\end{array}$ \\
\hline
\end{tabular}

Invima: Instituto Nacional de Vigilancia de Medicamentos y Alimentos.

* Clasificación K de movilidad esperada propuesta por Medicare, de Estados Unidos.

Anexo 2. Resultados esperados en salud, en la calidad de prestación de servicios y reducción de la inequidad según las intervenciones

\begin{tabular}{|c|c|c|c|}
\hline \multirow[b]{2}{*}{ Intervenciones } & \multicolumn{3}{|c|}{ Resultados esperados } \\
\hline & Resultados en salud & $\begin{array}{l}\text { Resultados en calidad de } \\
\text { la prestación de servicios } \\
\text { de salud }\end{array}$ & $\begin{array}{l}\text { Resultados en reducción de la } \\
\text { inequidad en salud }\end{array}$ \\
\hline $\begin{array}{l}\text { Se sugiere realizar la pletismografía, } \\
\text { junto con la presión arterial sistólica } \\
\text { digital y la presión arterial sistólica en } \\
\text { el tobillo, en caso de no contar con la } \\
\text { tensión transcutánea de oxígeno, en } \\
\text { pacientes mayores de } 16 \text { años con } \\
\text { alteraciones en la perfusión del pie que } \\
\text { van a ser sometidos a una amputación }\end{array}$ & $\begin{array}{l}\text { Definir el nivel más } \\
\text { distal posible de la } \\
\text { amputación. } \\
\text { Mejorar la cicatrización }\end{array}$ & $\begin{array}{l}\text { Servir como coadyuvante } \\
\text { para complementar el } \\
\text { examen del cirujano, } \\
\text { mejorando la precisión } \\
\text { diagnóstica y con ello } \\
\text { disminuir estancias } \\
\text { hospitalarias y } \\
\text { reintervenciones }\end{array}$ & $\begin{array}{l}\text { Favorece la equidad, porque se } \\
\text { puede aplicar a todas las personas, } \\
\text { independientemente de edad, sexo, } \\
\text { raza, religión y educación. } \\
\text { Al favorecer una adecuada curación, } \\
\text { disminuye la inequidad en ocupación, } \\
\text { pues permite un reintegro laboral } \\
\text { temprano. } \\
\text { Favorece la equidad en capital social, } \\
\text { ya que, al evitar reintervenciones, } \\
\text { permite que el paciente se haga } \\
\text { partícipe de la comunidad de forma } \\
\text { temprana. } \\
\text { No disminuye la inequidad en salud } \\
\text { en relación con el lugar de residencia, } \\
\text { porque la intervención no se realiza en } \\
\text { todos los niveles de atención del país }\end{array}$ \\
\hline $\begin{array}{l}\text { Se sugiere la realización de } \\
\text { procedimientos reconstructivos } \\
\text { de tejidos blandos, colgajos libres } \\
\text { o injertos, para el tratamiento de } \\
\text { defectos de cubrimiento de tejidos } \\
\text { blandos del muñón de amputación por } \\
\text { debajo de la rodilla, para conservar } \\
\text { esta articulación y mantener un nivel } \\
\text { de amputación transtibial }\end{array}$ & $\begin{array}{l}\text { Conservar articulación } \\
\text { de la rodilla y } \\
\text { mantener un nivel de } \\
\text { amputación transtibial. } \\
\text { Mejor recuperación } \\
\text { funcional de los } \\
\text { pacientes (entre más } \\
\text { longitud tenga la } \\
\text { extremidad, menos } \\
\text { demanda energética } \\
\text { existe para el paciente } \\
\text { al utilizar una prótesis). } \\
\text { Mejor adaptación } \\
\text { protésica y mejor } \\
\text { adherencia al uso de } \\
\text { la prótesis. } \\
\text { Adaptación protésica } \\
\text { con marcha sin } \\
\text { ayudas externas }\end{array}$ & $\begin{array}{l}\text { Baja frecuencia de } \\
\text { necesidad de ascenso del } \\
\text { muñón de amputación, } \\
\text { con pocas pérdidas de los } \\
\text { colgajos o los injertos }\end{array}$ & $\begin{array}{l}\text { Esta intervención favorece la equidad, } \\
\text { porque se puede aplicar a todas las } \\
\text { personas, independientemente de } \\
\text { edad, sexo, raza, religión y educación. } \\
\text { Disminuye la inequidad en ocupación, } \\
\text { al favorecer el reintegro laboral y la } \\
\text { independencia en actividades de la } \\
\text { vida diaria. } \\
\text { Al favorecer la adaptación protésica, } \\
\text { impacta en la inequidad por } \\
\text { discriminación. } \\
\text { Favorece la equidad en capital } \\
\text { social, ya que mejora la confianza } \\
\text { interpersonal entre los miembros de } \\
\text { una comunidad y la participación } \\
\text { cívica }\end{array}$ \\
\hline
\end{tabular}




\begin{tabular}{|c|c|}
\hline Intervenciones & Resultados en salud \\
\hline $\begin{array}{l}\text { Se sugiere realizar la reconstrucción de } \\
\text { la extremidad en pacientes con trauma } \\
\text { grave del miembro inferior, en lugar de } \\
\text { amputación, para mejorar la función y } \\
\text { disminuir los costos }\end{array}$ & $\begin{array}{l}\text { Mejora los aspectos } \\
\text { funcionales. } \\
\text { Mejora desenlaces en } \\
\text { el componente mental } \\
\text { y psicológico. } \\
\text { El dolor crónico } \\
\text { es menor en } \\
\text { los pacientes } \\
\text { reconstruidos en } \\
\text { comparación con los } \\
\text { pacientes llevados a } \\
\text { amputación }\end{array}$ \\
\hline
\end{tabular}

Se recomienda la amputación en dos tiempos, en lugar de la amputación en un solo tiempo con cierre primario, para disminuir el riesgo de infección del muñón, mejorar la cicatrización y acortar el tiempo de hospitalización

\section{Resultados esperados}

Resultados en calidad de
la prestación de servicios de salud Resultados en reducción de la
inequidad en salud

Disminución de costos Favorece la equidad, porque se para el sistema de salud a largo plazo, ya que los pacientes amputados necesitarían cambios

periódicos de la prótesis, Disminuye la inequidad en con los elevados costos que esto implica puede aplicar a todas las personas, independientemente de edad, sexo, raza, religión y educación. ocupación, al favorecer el reintegro laboral, debido a que la proporción de pacientes con discapacidad permanente es menor en el grupo de reconstrucción comparado con amputados (17,6 vs. $70 \%)$.

Favorece la equidad en capital social, ya que mejora la participación en sociedad.

Cuando el paciente conserva su extremidad, favorece la disminución de la inequidad en discriminación por discapacidad.

No disminuye la inequidad en salud en relación con el lugar de residencia, porque la intervención no se podría aplicar de igual manera en el territorio nacional (Ejemplo: un paciente con trauma grave. que vive distante a un hospital de alto nivel de complejidad de atención. no tendrá acceso a los servicios ofrecidos por este, entre ellos, cirugía plástica o vascular).

Disminuir el riesgo de Menor incidencia de las infección del muñón, complicaciones de la mejorar la cicatrización herida con dos tiempos y acortar el tiempo de quirúrgicos, entre ellos: hospitalización
Disminuye la inequidad por discriminación, pues al aplicarse la intervención, se reduce la posibilidad de transmitir la infección a otros y, por lo tanto, se merma la posibilidad de que el paciente permanezca en aislamiento.

intrahospitalarias

Reintervenciones

Hospitalización

prolongada

Aumento de costos
Favorece la equidad, porque se puede aplicar a todas las personas, independientemente de edad, sexo, raza, religión y educación.

Favorece la disminución de la inequidad en capital social, ya que al reducir las hospitalizaciones prolongadas, permite la participación cívica y la realización de los objetivos colectivos de la comunidad.

Favorece la disminución de la inequidad en ocupación, ya que al reducir la tasa de complicaciones, el paciente puede retornar de forma más pronta a sus actividades de la vida diaria y reintegrarse laboralmente 


\begin{tabular}{|c|c|c|c|}
\hline \multirow[b]{2}{*}{ Intervenciones } & \multicolumn{3}{|c|}{ Resultados esperados } \\
\hline & Resultados en salud & $\begin{array}{l}\text { Resultados en calidad de } \\
\text { la prestación de servicios } \\
\text { de salud }\end{array}$ & $\begin{array}{l}\text { Resultados en reducción de la } \\
\text { inequidad en salud }\end{array}$ \\
\hline $\begin{array}{l}\text { Se sugiere la analgesia epidural } \\
\text { perioperatoria, para disminuir el dolor } \\
\text { agudo del muñón y del miembro } \\
\text { fantasma en el periodo postoperatorio }\end{array}$ & $\begin{array}{l}\text { Disminuir el dolor } \\
\text { agudo del muñón en el } \\
\text { periodo postoperatorio }\end{array}$ & $\begin{array}{l}\text { Reducción en reconsultas } \\
\text { por dolor agudo } \\
\text { postoperatorio }\end{array}$ & $\begin{array}{l}\text { Disminuye la inequidad en capital } \\
\text { social, ya que al reducir el dolor, el } \\
\text { paciente puede reincorporarse más } \\
\text { tempranamente a la sociedad y } \\
\text { participar en ella, al igual que mejora } \\
\text { las relaciones dentro del entorno } \\
\text { familiar. } \\
\text { Disminuye la inequidad por } \\
\text { discriminación, pues al controlar } \\
\text { el dolor, la sociedad no genera } \\
\text { conductas que conduzcan al } \\
\text { aislamiento del paciente. } \\
\text { Favorece la disminución de la } \\
\text { inequidad en ocupación, pues al } \\
\text { reducir el dolor, el paciente puede } \\
\text { reincorporarse más tempranamente a } \\
\text { su vida laboral y a sus actividades de } \\
\text { la vida diaria. } \\
\text { Favorece la equidad, porque se } \\
\text { puede aplicar a todas las personas, } \\
\text { independientemente de edad, sexo, } \\
\text { raza, religión y educación }\end{array}$ \\
\hline $\begin{array}{l}\text { Se recomienda el uso de antibióticos } \\
\text { profilácticos, para la prevención de } \\
\text { infección del muñón }\end{array}$ & $\begin{array}{l}\text { Prevención de } \\
\text { infección del muñón }\end{array}$ & $\begin{array}{l}\text { Reduce los costos } \\
\text { asociados con su } \\
\text { tratamiento. } \\
\text { Disminución en proporción } \\
\text { de reamputación. } \\
\text { Menos días de estancia } \\
\text { hospitalaria. }\end{array}$ & $\begin{array}{l}\text { Favorece la equidad, porque se } \\
\text { puede aplicar a todas las personas, } \\
\text { independientemente de edad, sexo, } \\
\text { raza, religión y educación. } \\
\text { Favorece la disminución de la } \\
\text { inequidad en capital social, ya que } \\
\text { al reducir las hospitalizaciones } \\
\text { prolongadas, permite la participación } \\
\text { cívica y la realización de los objetivos } \\
\text { colectivos de la comunidad. } \\
\text { Favorece la disminución de la } \\
\text { inequidad en ocupación, ya que al } \\
\text { reducir reamputaciones, el paciente } \\
\text { puede retornar de forma más pronta } \\
\text { a sus actividades de la vida diaria y } \\
\text { reintegrarse laboralmente }\end{array}$ \\
\hline
\end{tabular}




\section{Resultados esperados}

Intervenciones

Se sugiere la amputación del mediopié o retropié en pacientes que tengan afectados dos o más rayos por causas isquémicas o por diabetes, para disminuir la frecuencia de reintervención para subir el nivel de la amputación
Se sugiere la realización de una amputación transfemoral en lugar de desarticulación de la rodilla en los pacientes que requieren una amputación del miembro inferior y no son candidatos a una por debajo de la rodilla, para disminuir las infecciones del muñón, mejorar la satisfacción cosmética con la prótesis, disminuir el dolor en el muñón de amputación y la necesidad de reamputación.
Disminuir las infecciones del muñón, mejorar la satisfacción cosmética con la prótesis, reducir el dolor en el muñón de amputación y la necesidad de reamputación

\section{Resultados en reducción de la inequidad en salud} de salud

Favorece la equidad, porque se puede aplicar a todas las personas, independientemente de edad, sexo, raza, religión y educación.

Disminuye la inequidad en ocupación, al reducir la posibilidad de reintervención, pues permite un reintegro laboral temprano.

Favorece la equidad en capital social, pues al evitar reintervenciones, permite que el paciente se haga partícipe de la comunidad de forma temprana.

Favorece la disminución en inequidad en las relaciones dependientes del tiempo, ya que al prevenir la realización de una reamputación, el paciente se puede integrar de forma más temprana socialmente

Disminuye la inequidad en discriminación por discapacidad, ya que los pacientes tienen una mejor percepción de la apariencia con su prótesis cuando se realiza la intervención.

Favorece la equidad, porque se puede aplicar a todas las personas, independientemente de edad, sexo raza, religión y educación.

Disminuye la inequidad en ocupación, al permitir un reintegro laboral, ya que reduce las infecciones y el dolor en el muñón.

Favorece la equidad en capital social, ya que al evitar reintervenciones y mejorar la satisfacción cosmética, la prótesis permite que el paciente se haga partícipe de la comunidad de forma temprana.

Favorece la disminución en inequidad en las relaciones dependientes del tiempo, ya que al prevenir la realización de una reamputación, el paciente se puede integrar de forma más temprana socialmente 


\begin{tabular}{|c|c|c|c|}
\hline \multirow[b]{2}{*}{ Intervenciones } & \multicolumn{3}{|c|}{ Resultados esperados } \\
\hline & Resultados en salud & $\begin{array}{c}\text { Resultados en calidad de } \\
\text { la prestación de servicios } \\
\text { de salud }\end{array}$ & $\begin{array}{l}\text { Resultados en reducción de la } \\
\text { inequidad en salud }\end{array}$ \\
\hline $\begin{array}{l}\text { Se recomienda la realización de } \\
\text { miodesis en el muñón de amputación, } \\
\text { para permitir un adecuado balance de } \\
\text { las fuerzas musculares en el muñón de } \\
\text { amputación y mejorar su estabilidad } \\
\text { dentro de la prótesis }\end{array}$ & $\begin{array}{l}\text { Permitir un adecuado } \\
\text { balance de las } \\
\text { fuerzas musculares } \\
\text { en el muñón de } \\
\text { amputación y mejorar } \\
\text { su estabilidad dentro } \\
\text { de la prótesis }\end{array}$ & $\begin{array}{l}\text { No costos adicionales } \\
\text { comparados con otras } \\
\text { técnicas quirúrgicas }\end{array}$ & $\begin{array}{l}\text { Disminuye la inequidad en } \\
\text { discriminación por discapacidad, ya } \\
\text { que los pacientes tienen una mejor } \\
\text { adaptación protésica. } \\
\text { Favorece la equidad, porque se } \\
\text { puede aplicar a todas las personas, } \\
\text { independientemente de edad, sexo, } \\
\text { raza, religión y educación- } \\
\text { Disminuye la inequidad en ocupación, } \\
\text { al permitir un reintegro laboral, } \\
\text { ya que la intervención mejora el } \\
\text { funcionamiento del muñón. } \\
\text { Favorece la equidad en capital } \\
\text { social, pues al mejorar la adaptación } \\
\text { protésica, el paciente se hace } \\
\text { partícipe de la comunidad de forma } \\
\text { temprana }\end{array}$ \\
\hline $\begin{array}{l}\text { Se recomienda, al realizar una } \\
\text { amputación transfemoral, obtener un } \\
\text { muñón óseo de por lo menos el } 57 \text { \% } \\
\text { del fémur contralateral, para aumentar } \\
\text { la velocidad y evitar la asimetría del } \\
\text { patrón de la marcha secundario a } \\
\text { alteraciones compensatorias en la } \\
\text { inclinación del tronco o de la pelvis }\end{array}$ & $\begin{array}{l}\text { Aumentar la velocidad } \\
\text { de la marcha y evitar } \\
\text { la asimetría del patrón } \\
\text { de esta, secundario } \\
\text { a alteraciones } \\
\text { compensatorias en la } \\
\text { inclinación del tronco o } \\
\text { de la pelvis }\end{array}$ & $\begin{array}{l}\text { Evitar deformidades en } \\
\text { abducción }\end{array}$ & $\begin{array}{l}\text { Disminuye la inequidad en } \\
\text { discriminación por discapacidad, ya } \\
\text { que los pacientes pueden presentar } \\
\text { deformidades. } \\
\text { Favorece la equidad, porque se } \\
\text { puede aplicar a todas las personas, } \\
\text { independientemente de edad, sexo, } \\
\text { raza, religión y educación. } \\
\text { Disminuye la inequidad en ocupación, } \\
\text { al permitir un reintegro laboral, ya que } \\
\text { mejora la marcha y su velocidad. } \\
\text { Favorece la equidad en capital social, } \\
\text { pues al mejorar la funcionalidad en la } \\
\text { marcha, el paciente puede participar } \\
\text { en sociedad }\end{array}$ \\
\hline $\begin{array}{l}\text { No se sugiere, de forma rutinaria, el } \\
\text { uso de sistemas cerrados de drenaje } \\
\text { por succión después del cierre } \\
\text { definitivo en pacientes que requieran } \\
\text { una amputación del miembro inferior, } \\
\text { para disminuir el riesgo de infección y } \\
\text { necesidad de cirugías adicionales por } \\
\text { hematomas o seromas }\end{array}$ & $\begin{array}{l}\text { Disminuir el riesgo de } \\
\text { infección y necesidad } \\
\text { de cirugías adicionales } \\
\text { por hematomas o } \\
\text { seromas }\end{array}$ & $\begin{array}{l}\text { Disminución de estancias } \\
\text { hospitalarias, al reducir } \\
\text { tasas de complicaciones } \\
\text { posoperatorias } \\
\text { (hematomas, infección, } \\
\text { reintervenciones) }\end{array}$ & $\begin{array}{l}\text { Favorece la equidad, porque se } \\
\text { puede aplicar a todas las personas, } \\
\text { independientemente de edad, sexo, } \\
\text { raza, religión y educación. } \\
\text { Disminuye la inequidad en ocupación, } \\
\text { al permitir un reintegro laboral, ya que } \\
\text { reduce la estancia hospitalaria. } \\
\text { Favorece la equidad en capital social, } \\
\text { ya que al disminuir la necesidad de } \\
\text { procedimientos quirúrgicos y de } \\
\text { estancias hospitalarias, el paciente } \\
\text { puede reincorporarse a la sociedad y } \\
\text { participar en ella }\end{array}$ \\
\hline
\end{tabular}




\section{Resultados esperados}

\begin{tabular}{l}
\multicolumn{1}{c}{ Intervenciones } \\
\hline Tratamiento farmacológico, para el \\
tratamiento del dolor neuropático. \\
Se recomienda, en primer lugar, la \\
pregabalina, seguida de gabapentina, \\
amitriptilina y duloxetina como \\
monoterapia
\end{tabular}

\section{Resultados en salud}

Mejorar la baja en la
intensidad el dolor
neuropático

neuropático

monoterapia
Se sugiere el uso de una prótesis inmediata postoperatoria, para mejorar la remodelación del muñón, facilitar la adaptación protésica y el funcionamiento, y disminuir el tiempo para la adaptación protésica definitiva

\section{Mejorar la}

remodelación del

muñón, facilitar la adaptación protésica y el funcionamiento humano, y disminuir el tiempo para la adaptación protésica definitiva

\section{Resultados en reducción de la inequidad en salud}

\section{Reducción en reconsultas Disminuye la inequidad en} por dolor

discriminación por discapacidad, al generar una satisfacción en el paciente con el uso de la prótesis.

Favorece la equidad, porque se puede aplicar a todas las personas, independientemente de edad, sexo, raza, religión y educación.

Disminuye la inequidad en ocupación, ya que una adecuada adaptación protésica permite que el paciente se reintegre laboralmente.

Favorece la equidad en capital social, ya que al disminuir la necesidad de reconsultas, el paciente puede participar en sociedad.

Favorece la disminución de la inequidad en ocupación, pues al reducir el dolor, el paciente puede reincorporarse más tempranamente a su vida laboral y a sus actividades de la vida diaria

Disminuir estancia hospitalaria

Disminuye la inequidad en discriminación por discapacidad, ya que los pacientes se visualizan de forma inmediata con su prótesis.

Favorece la equidad, porque se puede aplicar a todas las personas, independientemente de edad, sexo, raza, religión y educación.

Disminuye la inequidad en ocupación, al permitir un reintegro laboral, ya que hay una adaptación protésica precoz y reduce la estancia hospitalaria.

Favorece la equidad en capital social, ya que al disminuir la necesidad de procedimientos quirúrgicos y de estancias hospitalarias, el paciente puede reincorporarse a la sociedad y participar en ella 


\begin{tabular}{|c|c|c|c|}
\hline \multirow[b]{2}{*}{ Intervenciones } & \multicolumn{3}{|c|}{ Resultados esperados } \\
\hline & Resultados en salud & $\begin{array}{l}\text { Resultados en calidad de } \\
\text { la prestación de servicios } \\
\text { de salud }\end{array}$ & $\begin{array}{l}\text { Resultados en reducción de la } \\
\text { inequidad en salud }\end{array}$ \\
\hline $\begin{array}{l}\text { Se recomienda, en las personas con } \\
\text { amputación por encima o debajo de } \\
\text { la rodilla, la adaptación de un pie tipo } \\
\text { sach, para mejorar la función de la } \\
\text { marcha. }\end{array}$ & $\begin{array}{l}\text { Mejorar la función de } \\
\text { la marcha }\end{array}$ & $\begin{array}{l}\text { Es costoefectivo para } \\
\text { pacientes con bajo nivel } \\
\text { de actividad }\end{array}$ & $\begin{array}{l}\text { Disminuye la inequidad en } \\
\text { discriminación por discapacidad, ya } \\
\text { que la adaptación de un pie protésico } \\
\text { permite una aceptación personal de la } \\
\text { nueva condición. }\end{array}$ \\
\hline \multirow[t]{3}{*}{$\begin{array}{l}\text { En pacientes adultos mayores con } \\
\text { nivel funcional } \mathrm{K} 1^{*} \text { que hayan utilizado } \\
\text { previamente un pie tipo sach podría } \\
\text { evaluarse la posibilidad de adaptación } \\
\text { de un pie articulado }\end{array}$} & & & $\begin{array}{l}\text { Favorece la equidad, porque se } \\
\text { puede aplicar a todas las personas, } \\
\text { independientemente de edad, sexo, } \\
\text { raza, religión y educación. }\end{array}$ \\
\hline & & & $\begin{array}{l}\text { Disminuye la inequidad en ocupación, } \\
\text { al posibilitar el reintegro laboral, ya } \\
\text { que la adaptación de un pie protésico } \\
\text { permite mejorar la función para la } \\
\text { marcha. }\end{array}$ \\
\hline & & & $\begin{array}{l}\text { Favorece la equidad en capital social, } \\
\text { pues al mejorar la marcha, el paciente } \\
\text { puede reincorporarse a la sociedad y } \\
\text { participar en ella }\end{array}$ \\
\hline \multirow{4}{*}{$\begin{array}{l}\text { Se sugiere la adaptación de un } \\
\text { pie articulado o uno de respuesta } \\
\text { dinámica en personas con } \\
\text { requerimientos mayores de actividad. } \\
\text { Recomendado por un médico } \\
\text { especialista con entrenamiento en el } \\
\text { área de prótesis y que las condiciones } \\
\text { sociales o ambientales lo posibiliten }\end{array}$} & $\begin{array}{l}\text { Mejorar la función de } \\
\text { la marcha }\end{array}$ & $\begin{array}{l}\text { Es costoefectivo para } \\
\text { pacientes con mayor nivel } \\
\text { de actividad }\end{array}$ & $\begin{array}{l}\text { Disminuye la inequidad en } \\
\text { discriminación por discapacidad, ya } \\
\text { que la adaptación de un pie protésico } \\
\text { permite una aceptación personal de la } \\
\text { nueva condición. }\end{array}$ \\
\hline & & & $\begin{array}{l}\text { Favorece la equidad, porque se } \\
\text { puede aplicar a todas las personas, } \\
\text { independientemente de edad, sexo, } \\
\text { raza, religión y educación. }\end{array}$ \\
\hline & & & $\begin{array}{l}\text { Disminuye la inequidad en ocupación, } \\
\text { al posibilitar el reintegro laboral, ya } \\
\text { que la adaptación de un pie protésico } \\
\text { permite mejorar la función para la } \\
\text { marcha. }\end{array}$ \\
\hline & & & $\begin{array}{l}\text { Favorece la equidad en capital } \\
\text { social, ya que al mejorar la marcha, } \\
\text { el paciente puede reincorporarse a la } \\
\text { sociedad y participar en ella }\end{array}$ \\
\hline
\end{tabular}




\section{Resultados esperados}

\begin{tabular}{|c|c|c|c|}
\hline \multirow[b]{2}{*}{ Intervenciones } & \\
\hline & Resultados en salud & $\begin{array}{l}\text { Resultados en calidad de } \\
\text { la prestación de servicios } \\
\text { de salud }\end{array}$ & $\begin{array}{l}\text { Resultados en reducción de la } \\
\text { inequidad en salud }\end{array}$ \\
\hline $\begin{array}{l}\text { Se sugiere la adaptación de una } \\
\text { prótesis con cuenca de contacto total y } \\
\text { funda de silicona en las personas con } \\
\text { amputación por debajo de la rodilla, } \\
\text { de acuerdo con las condiciones del } \\
\text { muñón y las habilidades residuales del } \\
\text { paciente para mejorar la función de la } \\
\text { marcha }\end{array}$ & $\begin{array}{l}\text { Mejorar la función de } \\
\text { la marcha }\end{array}$ & $\begin{array}{l}\text { Disminuir el número de } \\
\text { reconsultas relacionadas } \\
\text { con problemas de la } \\
\text { adaptación protésica }\end{array}$ & $\begin{array}{l}\text { Disminuye la inequidad en } \\
\text { discriminación por discapacidad, } \\
\text { al generar una satisfacción en el } \\
\text { paciente con el uso de la prótesis. } \\
\text { Favorece la equidad, porque se } \\
\text { puede aplicar a todas las personas, } \\
\text { independientemente de edad, sexo, } \\
\text { raza, religión y educación. } \\
\text { Disminuye la inequidad en ocupación, } \\
\text { ya que una adecuada adaptación } \\
\text { protésica permite que el paciente se } \\
\text { reintegre laboralmente. } \\
\text { Favorece la equidad en capital social, } \\
\text { ya que al disminuir la necesidad } \\
\text { de reconsultas, el paciente puede } \\
\text { participar en sociedad. }\end{array}$ \\
\hline $\begin{array}{l}\text { Se sugiere, en las personas con } \\
\text { amputación por debajo de rodilla, una } \\
\text { prótesis con cuenca de contacto total, } \\
\text { con encaje de interface en silicona, } \\
\text { copolímero o poliuretano. El uso de } \\
\text { una válvula de vacío o un pin con traba } \\
\text { y lanzadera debe ser individualizado } \\
\text { teniendo en cuenta las condiciones } \\
\text { físicas del paciente y el muñón, el nivel } \\
\text { de actividad y su ámbito personal, } \\
\text { social y ambiental }\end{array}$ & $\begin{array}{l}\text { Menor desplazamiento } \\
\text { vertical del muñón } \\
\text { dentro del encaje o } \\
\text { dentro de la cuenca. } \\
\text { Evitar sonidos } \\
\text { indeseables. } \\
\text { Que sea segura y fácil } \\
\text { de poner y quitar }\end{array}$ & $\begin{array}{l}\text { Disminuir el número de } \\
\text { reconsultas relacionadas } \\
\text { con problemas de la } \\
\text { adaptación protésica }\end{array}$ & $\begin{array}{l}\text { Disminuye la inequidad en } \\
\text { discriminación por discapacidad, } \\
\text { al generar una satisfacción en el } \\
\text { paciente con el uso de la prótesis. } \\
\text { Favorece la equidad, porque se } \\
\text { puede aplicar a todas las personas, } \\
\text { independientemente de edad, sexo, } \\
\text { raza, religión y educación. } \\
\text { Disminuye la inequidad en ocupación, } \\
\text { ya que una adecuada adaptación } \\
\text { protésica permite que el paciente se } \\
\text { reintegre laboralmente. } \\
\text { Favorece la equidad en capital social, } \\
\text { ya que al disminuir la necesidad } \\
\text { de reconsultas, el paciente puede } \\
\text { participar en sociedad. }\end{array}$ \\
\hline
\end{tabular}




\begin{tabular}{|c|c|c|c|}
\hline \multirow[b]{2}{*}{ Intervenciones } & \multicolumn{3}{|c|}{ Resultados esperados } \\
\hline & Resultados en salud & $\begin{array}{c}\text { Resultados en calidad de } \\
\text { la prestación de servicios } \\
\text { de salud }\end{array}$ & $\begin{array}{l}\text { Resultados en reducción de la } \\
\text { inequidad en salud }\end{array}$ \\
\hline $\begin{array}{l}\text { Se sugiere, en las personas con } \\
\text { amputación por encima de la rodilla } \\
\text { y un nivel esperado de actividad } \\
\text { K1, }{ }^{*} \text { la adaptación de una rodilla } \\
\text { monocéntrica con bloqueo manual o } \\
\text { con freno de carga; en los K2, K3 y } \\
\text { K4, }{ }^{\star} \text { una monocéntrica o policéntrica } \\
\text { de control de fluidos, para mejorar la } \\
\text { función de la marcha en los primeros } \\
12 \text { meses luego de la amputación }\end{array}$ & $\begin{array}{l}\text { Mejorar la función } \\
\text { de la marcha en } \\
\text { los primeros } 12 \\
\text { meses luego de la } \\
\text { amputación }\end{array}$ & $\begin{array}{l}\text { Adaptaciones protésicas } \\
\text { exitosas de acuerdo } \\
\text { con las necesidades del } \\
\text { paciente y así no aumentar } \\
\text { costos }\end{array}$ & $\begin{array}{l}\text { Disminuye la inequidad en } \\
\text { discriminación por discapacidad, } \\
\text { al generar una satisfacción en el } \\
\text { paciente con el uso de la prótesis. } \\
\text { Favorece la equidad, porque se } \\
\text { puede aplicar a todas las personas, } \\
\text { independientemente de edad, sexo, } \\
\text { raza, religión y educación. } \\
\text { Disminuye la inequidad en ocupación, } \\
\text { ya que una adecuada adaptación } \\
\text { protésica permite que el paciente se } \\
\text { reintegre laboralmente. } \\
\text { Favorece la equidad en capital social, } \\
\text { ya que al disminuir la necesidad } \\
\text { de reconsultas, el paciente puede } \\
\text { participar en sociedad y facilitar la } \\
\text { realización de los objetivos colectivos } \\
\text { de la comunidad y la fortaleza de sus } \\
\text { conexiones políticas, que pueden } \\
\text { facilitar el acceso a los servicios }\end{array}$ \\
\hline $\begin{array}{l}\text { Se sugiere, en las personas mayores } \\
\text { de } 16 \text { años con desarticulación } \\
\text { de rodilla y un nivel esperado de } \\
\text { actividad K1, la adaptación de una } \\
\text { rodilla policéntrica mecánica para } \\
\text { desarticulado de rodilla; y en los K2, } \\
\text { K3 y K4, una policéntrica de control de } \\
\text { fluidos, para desarticulación de rodilla, } \\
\text { para mejorar la función de la marcha } \\
\text { en los primeros } 12 \text { meses luego de la } \\
\text { amputación }\end{array}$ & $\begin{array}{l}\text { Mejorar la función } \\
\text { de la marcha en } \\
\text { los primeros } 12 \\
\text { meses luego de la } \\
\text { amputación }\end{array}$ & $\begin{array}{l}\text { Adaptaciones protésicas } \\
\text { exitosas de acuerdo } \\
\text { con las necesidades del } \\
\text { paciente y así no aumentar } \\
\text { costos }\end{array}$ & $\begin{array}{l}\text { Disminuye la inequidad en } \\
\text { discriminación por discapacidad, } \\
\text { al generar una satisfacción en el } \\
\text { paciente con el uso de la prótesis. } \\
\text { Favorece la equidad, porque se } \\
\text { puede aplicar a todas las personas, } \\
\text { independientemente de edad, sexo, } \\
\text { raza, religión y educación. } \\
\text { Disminuye la inequidad en ocupación, } \\
\text { ya que una adecuada adaptación } \\
\text { protésica permite que el paciente se } \\
\text { reintegre laboralmente. } \\
\text { Favorece la equidad en capital social, } \\
\text { ya que al disminuir la necesidad } \\
\text { de reconsultas, el paciente puede } \\
\text { participar en sociedad }\end{array}$ \\
\hline
\end{tabular}




\section{Resultados esperados}

Intervenciones

Se recomienda, en las personas con amputación por encima de la rodilla y niveles moderados o altos de actividad, la adaptación de alguna de las variantes de cuenca de contenimiento isquiático, para mejorar la función de la marcha. Se recomienda, en las personas con niveles bajos de actividad, la adaptación de una cuenca de tipo cuadrilateral

Se recomienda la adaptación individualizada de un sistema de suspensión acorde con las capacidades funcionales del paciente y la condición del muñón.

Para pacientes con niveles bajos de actividad (K1 y K2), se utilizan encajes de silicona con sujeción mediante correaje y velcro, o sujeción mediante cordón.

En pacientes muy activos (K3 y K4), se prefiere los sistemas de vacío, pues crean mejor confort, mejor sujeción y son más fáciles de adaptar por el paciente que los de pin y lanzadera

\section{Resultados en reducción de la inequidad en salud}

\section{Mejorar la función de Adaptaciones protésicas Disminuye la inequidad en}

la marcha

exitosas de acuerdo

discriminación por discapacidad, con las necesidades del al generar una satisfacción en el paciente y así no aumentar paciente con el uso de la prótesis. costos

Favorece la equidad, porque se puede aplicar a todas las personas, independientemente de edad, sexo, raza, religión y educación.

Disminuye la inequidad en ocupación, ya que una adecuada adaptación protésica permite que el paciente se reintegre laboralmente.

Favorece la equidad en capital social, ya que al disminuir la necesidad de reconsultas, el paciente puede participar en sociedad

Mejor confort, mejor sujeción
Adaptaciones protésicas exitosas de acuerdo con las necesidades del paciente y así no aumentar costos
Disminuye la inequidad en discriminación por discapacidad, al generar una satisfacción en el paciente con el uso de la prótesis.

Favorece la equidad, porque se puede aplicar a todas las personas, independientemente de edad, sexo, raza, religión y educación.

Disminuye la inequidad en ocupación, ya que una adecuada adaptación protésica permite que el paciente se reintegre laboralmente.

Favorece la equidad en capital social, ya que al disminuir la necesidad de reconsultas, el paciente puede participar en sociedad 


\begin{tabular}{|c|c|c|c|}
\hline \multirow[b]{2}{*}{ Intervenciones } & \multicolumn{3}{|c|}{ Resultados esperados } \\
\hline & Resultados en salud & $\begin{array}{c}\text { Resultados en calidad de } \\
\text { la prestación de servicios } \\
\text { de salud }\end{array}$ & $\begin{array}{l}\text { Resultados en reducción de la } \\
\text { inequidad en salud }\end{array}$ \\
\hline $\begin{array}{l}\text { Se recomienda la implementación } \\
\text { de un programa de rehabilitación } \\
\text { física que incluya fuerza muscular, } \\
\text { movilidad articular, equilibrio, marcha } \\
\text { y reacondicionamiento físico, en } \\
\text { pacientes con amputación de miembro } \\
\text { inferior por causas traumáticas, } \\
\text { vasculares o por diabetes, para } \\
\text { mejorar la marcha, la movilidad y la } \\
\text { adaptación protésica }\end{array}$ & $\begin{array}{l}\text { Mejorar la marcha, } \\
\text { la movilidad y la } \\
\text { adaptación protésica }\end{array}$ & $\begin{array}{l}\text { Disminuir reconsultas } \\
\text { por motivos asociados a } \\
\text { la rehabilitación, como: } \\
\text { dificultades en traslados } \\
\text { y trasferencias, dolor, } \\
\text { necesidad de ayudas de } \\
\text { asistencia }\end{array}$ & $\begin{array}{l}\text { Favorece la equidad, porque se } \\
\text { puede aplicar a todas las personas, } \\
\text { independientemente de edad, sexo, } \\
\text { raza, religión y educación. } \\
\text { Disminuye la inequidad en ocupación, } \\
\text { ya que al mejorar la fuerza, la marcha } \\
\text { favorece el retorno de los pacientes } \\
\text { a sus actividades de la vida diaria y } \\
\text { laborales. } \\
\text { Disminuye la inequidad en capital } \\
\text { social, ya que al mejorar su condición } \\
\text { física, el paciente puede participar en } \\
\text { sociedad. } \\
\text { No disminuye la inequidad en salud } \\
\text { en relación con el lugar de residencia, } \\
\text { ya que no todos los pacientes tienen } \\
\text { acceso a lugares que cuenten con } \\
\text { terapeutas físicos }\end{array}$ \\
\hline $\begin{array}{l}\text { Se recomienda la rehabilitación } \\
\text { ocupacional y las adaptaciones } \\
\text { ergonómicas en pacientes a quienes } \\
\text { les fue amputado un miembro inferior, } \\
\text { para mejorar el funcionamiento y } \\
\text { facilitar el retorno al trabajo o a una } \\
\text { ocupación }\end{array}$ & $\begin{array}{l}\text { Mejorar el } \\
\text { funcionamiento y } \\
\text { facilitar el retorno } \\
\text { al trabajo o a una } \\
\text { ocupación }\end{array}$ & $\begin{array}{l}\text { Disminuir el número } \\
\text { de consultas por } \\
\text { molestias relacionadas } \\
\text { con el sistema } \\
\text { musculoesquelético }\end{array}$ & $\begin{array}{l}\text { Favorece la equidad, porque se } \\
\text { puede aplicar a todas las personas, } \\
\text { independientemente de edad, sexo, } \\
\text { raza, religión y educación. } \\
\text { Disminuye la inequidad en ocupación, } \\
\text { pues el desarrollo de adaptaciones } \\
\text { facilita el desempeño por parte del } \\
\text { paciente en el ámbito laboral, como } \\
\text { en sus actividades de la vida diaria. } \\
\text { Disminuye la inequidad en } \\
\text { capital social, pues al mejorar su } \\
\text { desempeño, el paciente puede } \\
\text { participar de forma más activa en } \\
\text { sociedad. } \\
\text { No disminuye la inequidad en salud } \\
\text { en relación con el lugar de residencia, } \\
\text { ya que no todos los pacientes tienen } \\
\text { acceso a lugares que cuenten con } \\
\text { terapeutas ocupacionales }\end{array}$ \\
\hline
\end{tabular}




\section{Resultados esperados}

Intervenciones

Se recomienda la implementación de un proceso de rehabilitación integral: cardiopulmonar, musculoesquelético, psicosocial, actividades de la vida diaria y para el trabajo, en pacientes con amputación de miembro inferior, de causas traumáticas, vasculares o por diabetes, para mejorar o mantener el funcionamiento, la independencia en las actividades de la vida diaria, el reintegro y la participación social

Se sugiere la utilización de la Escala de Houghton para evaluar la adaptación protésica
Realizar un seguimiento de la adaptación protésica

\section{Resultados en calidad de la prestación de servicios de salud}

\section{Resultados en reducción de la} inequidad en salud

\section{Disminuir el número de} consultas por molestias relacionadas sea con lo físico, lo mental o lo social

.

\section{actividades de la vida diaria, el reintegro y la participación social \\ Mejorar o mantener \\ el funcionamiento, la

participación social

Favorece la equidad, porque se raza, religión y educación. puede aplicar a todas las personas, independientemente de edad, sexo,

Disminuye la inequidad en ocupación, ya que al fortalecer todos los ámbitos de la vida (físico, mental, social), el paciente podrá reincorporarse más fácil a su vida cotidiana

Disminuye la inequidad en capital social, ya que al mejorar todas las áreas del ser, el paciente podrá participar de forma más activa en sociedad.

No disminuye la inequidad en salud en relación con el lugar de residencia, ya que no todos los pacientes tienen acceso a lugares que cuenten con psicólogos, terapeutas físicos y ocupacionales

No genera un incremento Favorece la equidad, porque se sustancial en los costos puede aplicar a todas las personas, de su aplicación puede aplicar a todas las personas,
independientemente de edad, sexo, raza, religión y educación.

Disminuye la inequidad en ocupación y capital social, ya que al ser aplicada la intervención, pueden realizarse los ajustes correspondientes para mejorar la adaptación protésica y así mejorar la independencia en actividades de la vida diaria, desempeño laboral y participación en la comunidad

\footnotetext{
* Clasificación K de movilidad esperada propuesta por Medicare, de Estados Unidos.
} 